\title{
Computer Vision, Machine Learning, and the Promise of Phenomics in Ecology and Evolutionary Biology
}

\author{
Moritz D. Lürig ${ }^{1 *}$, Seth Donoughe ${ }^{2}$, Erik I. Svensson ${ }^{1}$, Arthur Porto ${ }^{3,4}$ and \\ Masahito Tsuboi ${ }^{1}$ \\ ' Department of Biology, Lund University, Lund, Sweden, ${ }^{2}$ Department of Molecular Genetics and Cell Biology, University \\ of Chicago, Chicago, IL, United States, ${ }^{3}$ Department of Biological Sciences, Louisiana State University, Baton Rouge, LA, \\ United States, ${ }^{4}$ Center for Computation and Technology, Louisiana State University, Baton Rouge, LA, United States
}

OPEN ACCESS

Edited by:

Aurore Ponchon

University of Aberdeen,

United Kingdom

Reviewed by:

Stefan Schneider,

University of Guelph, Canada

Methun Kamruzzaman,

University of Virginia, United States

*Correspondence: Moritz D. Lürig

moritz.lurig@biol.lu.se

Specialty section:

This article was submitted to Behavioral and Evolutionary Ecology,

a section of the journal

Frontiers in Ecology and Evolution

Received: 16 December 2020

Accepted: 22 February 2021

Published: 21 April 2021

Citation:

Lürig MD, Donoughe $S$, Svensson El, Porto A and Tsuboi M (2021) Computer Vision, Machine Learning, and the Promise of Phenomics in Ecology and Evolutionary Biology.

Front. Ecol. Evol. 9:642774. doi: 10.3389/fevo.2021.642774
For centuries, ecologists and evolutionary biologists have used images such as drawings, paintings and photographs to record and quantify the shapes and patterns of life. With the advent of digital imaging, biologists continue to collect image data at an ever-increasing rate. This immense body of data provides insight into a wide range of biological phenomena, including phenotypic diversity, population dynamics, mechanisms of divergence and adaptation, and evolutionary change. However, the rate of image acquisition frequently outpaces our capacity to manually extract meaningful information from images. Moreover, manual image analysis is low-throughput, difficult to reproduce, and typically measures only a few traits at a time. This has proven to be an impediment to the growing field of phenomics - the study of many phenotypic dimensions together. Computer vision (CV), the automated extraction and processing of information from digital images, provides the opportunity to alleviate this longstanding analytical bottleneck. In this review, we illustrate the capabilities of CV as an efficient and comprehensive method to collect phenomic data in ecological and evolutionary research. First, we briefly review phenomics, arguing that ecologists and evolutionary biologists can effectively capture phenomic-level data by taking pictures and analyzing them using CV. Next we describe the primary types of image-based data, review CV approaches for extracting them (including techniques that entail machine learning and others that do not), and identify the most common hurdles and pitfalls. Finally, we highlight recent successful implementations and promising future applications of CV in the study of phenotypes. In anticipation that CV will become a basic component of the biologist's toolkit, our review is intended as an entry point for ecologists and evolutionary biologists that are interested in extracting phenotypic information from digital images.

Keywords: computer vision, machine learning, phenomics, high-throughput phenotyping, high-dimensional data, image analysis, image segmentation, measurement theory

\section{FROM PHENOTYPES TO PHENOMICS}

Faced with the overwhelming complexity of the living world, most life scientists confine their efforts to a small set of observable traits. Although a drastic simplification of organismal complexity, the focus on single phenotypic attributes often provides a tractable, operational approach to understand biological phenomena, e.g., phenotypic trait diversity, population dynamics, mechanisms of 
divergence, and adaptation and evolutionary change. However, there are also obvious limitations in how much we can learn from studying small numbers of phenotypes in isolation. Evolutionary and conservation biologist Michael Soulé was one of the first to demonstrate the value of collecting and analyzing many phenotypes at once in his early study of the side-blotched lizard [Uta stansburiana (Soulé, 1967)]; reviewed in Houle et al. (2010). While doing so, he defined the term "phenome" as "the phenotype as a whole" (Soulé, 1967). Phenomics, by extension, is the comprehensive study of phenomes. In practice, this entails collecting and analyzing multidimensional phenotypes with a wide range of quantitative and high-throughput methods (Bilder et al., 2009). Given that biologists are now attempting to understand increasingly complex and high dimensional relationships between traits (Walsh, 2007), it is surprising that phenomics still remains underutilized (Figure 1), both as methodological approach and as an overarching conceptual and analytical framework (Houle et al., 2010).

Phenomic datasets are essential if we are to understand some of the most compelling but challenging questions in the study of ecology and evolution. For instance, phenotypic diversity can fundamentally affect population dynamics (Laughlin et al., 2020), community assembly (Chesson, 2000), and the functioning and stability of ecosystems (Hooper et al., 2005). Such functional diversity (Petchey and Gaston, 2006) is ecologically extremely relevant, but can be hard to quantify exactly, because organisms interact with their environment through many traits of which a large portion would need to be measured (Villéger et al., 2008; Blonder, 2018). Moreover, natural selection typically does not operate on single traits, but on multiple traits simultaneously (Lande and Arnold, 1983; Phillips and Arnold, 1999), which can lead to correlations (Schluter, 1996; Sinervo and Svensson, 2002; Svensson et al., 2021) and pleiotropic relationships between genes (Visscher and Yang, 2016; Saltz et al., 2017). Phenotypic plasticity, which is increasingly recognized in mediating evolutionary trajectories (Pfennig et al., 2010), is also an inherently multivariate phenomenon involving many traits and interactions between traits, so it should be quantified as such (Morel-Journel et al., 2020). Put simply: if we are to draw a complete picture of biological processes and aim to understand their causal relationships at various levels of biological organization, we need to measure more traits, from more individuals and a wider range of different species.

High dimensional phenotypic data are also needed for uncovering the causal links between genotypes, environmental factors, and phenotypes, i.e., to understand the genotypephenotype map (Houle et al., 2010; Orgogozo et al., 2015). The advent of genomics - high throughput molecular methods to analyze the structure, function or evolution of an organism's genome in parts or as a whole (Church and Gilbert, 1984; Feder and Mitchell-Olds, 2003) - has already improved our understanding of many biological phenomena. This includes the emergence and maintenance of biological diversity (Seehausen et al., 2014), the inheritance and evolution of complex traits (Pitchers et al., 2019), and the evolutionary origin of key metabolic traits (Ishikawa et al., 2019). Thus, accessible molecular tools have lowered the hurdles for discovery-based genomic research and shifted the focus away from the study of observable organismal traits and phenotypes toward their molecular basis. However, a similar "moonshot-program" for the phenotype, i.e., an ensemble of phenomics methods that matches genomics in their comprehensiveness, is still lacking (Freimer and Sabatti, 2003). The growing mismatch in how efficiently molecular and phenotypic data are collected may hamper further scientific progress in ecological and evolutionary research (Houle et al., 2010; Orgogozo et al., 2015; Lamichhaney et al., 2019).

Following previous calls for phenomic research programs (Bilder et al., 2009; Houle et al., 2010; Furbank and Tester, 2011), some recent studies have collected phenotypic data with high dimensionality and on a massive scale, for example, in plants (Ubbens and Stavness, 2017), animals (Cheng et al., 2011; Kühl and Burghardt, 2013; Pitchers et al., 2019), and microbes (Zackrisson et al., 2016; French et al., 2018). All of these studies use some form of image analysis to quantify external (i.e., morphology or texture) and internal phenotypes (e.g., cells, bones or tissue), or behavioral phenotypes and biomechanical properties (e.g., body position, pose or movement). Such data represent phenomics in a narrow sense: the collection of (external, internal, and behavioral) phenotypic data on an organism-wide scale (Houle et al., 2010). In addition, many biologists also use image analysis to detect presence and absence of organisms (e.g., within a population, community or environment; e.g., by means of camera traps or satellite images), or to identify species (by experts or algorithms). While species monitoring and taxonomic identification constitutes an important and rapidly growing discipline on its own (Norouzzadeh et al., 2018; Wäldchen and Mäder, 2018; Høye et al., 2020), this review focuses on the extraction of phenotypic data from digital images as a key methodological approach for the study of phenomes (Houle et al., 2010).

Previous work has supplied us with an immense body of image data that has provided insight into a wide range of biological phenomena, yet when biologists manually extract phenotypes from images for phenomic-scale research, they confront several main bottlenecks (Houle et al., 2003; Gerum et al., 2017; Ubbens and Stavness, 2017). A major constraint when working with large amounts of images $(\sim 1,000$ or more) is processing time and cost. Manual extraction of phenotypic data from images is slow and it requires trained domain experts whose work is extremely expensive. Moreover, the collection of such metrics in a manual fashion entails subjective decisions by the researcher, which may make it prone to error, and certainly makes reproducibility difficult. Last, manually measured traits tend to be low-dimensional measurements of higher dimensional traits. For example, external color traits, such as human eye color phenotypes, are often scored as discrete categories (e.g., brown vs. blue phenotypes), whereas pixel level information (number of brown vs. blue pixels) can provide a continuous phenotypic metric (Liu et al., 2010). Such quantitative, high-dimensional data can provide insight into previously hidden axes of variation, and may help provide a mechanistic understanding of the interplay of phenotypes, their genetic underpinnings, and the environment.

In this review we extol computer vision (CV; for a definition of terms in italics see see Box 1), the automatic extraction of 

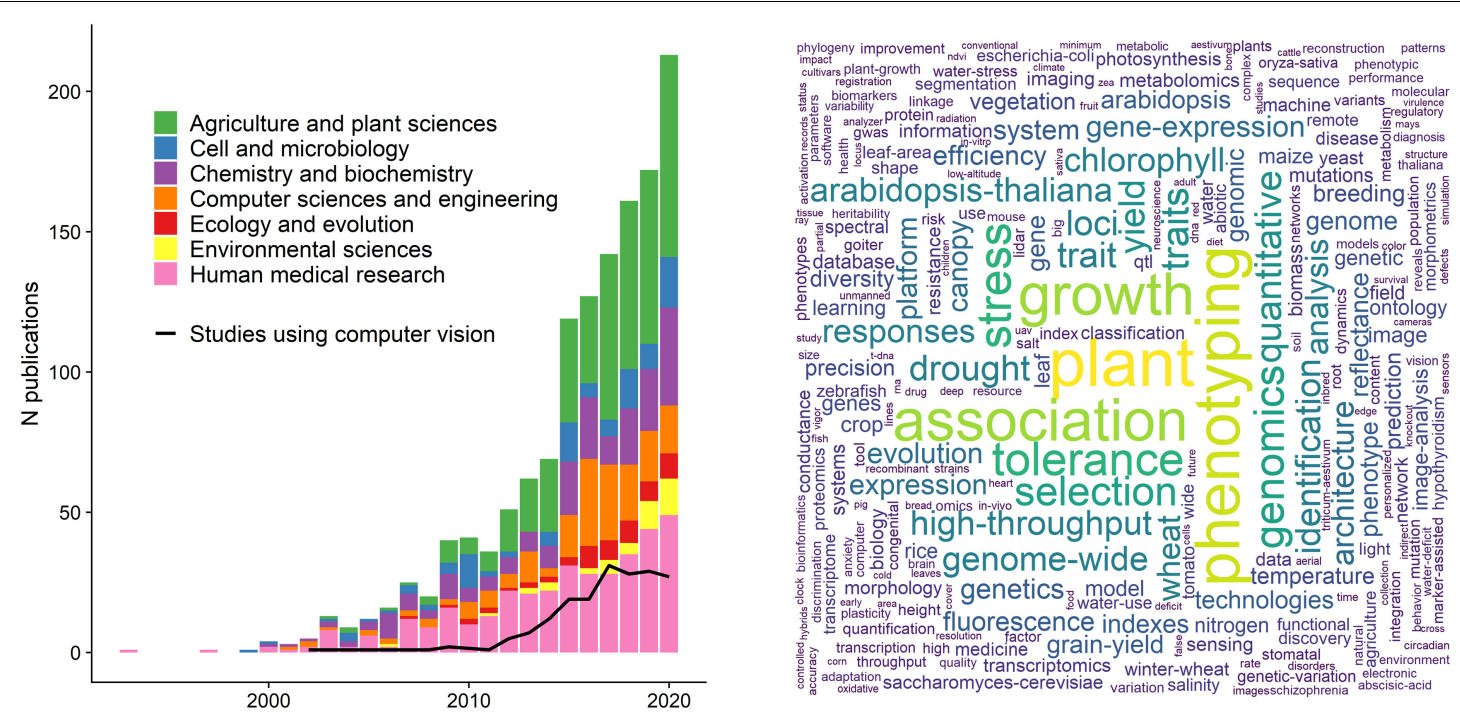

FIGURE 1 | The current state of phenomics research. Left panel: a literature survey using the search key "phenomic*" in a Web of Science topic search (title, keywords, abstract) resulted in 1,408 papers (01/03/2021). Here we show only papers published between 1990 and before 2021 (1,343 papers) for better visual inference. Traditionally, phenomics approaches are used in agricultural sciences and crop research to maximize yield, as well as in human medicine to study drug responses and disorder phenotypes. The black line denotes the studies that used computer vision or some sort of image analysis (acquired with a topic search among the retrieved papers using the strings "computer vision," "image analysis," "image based," "image processing"), indicating that only a small subset of the studies uses image analysis. Right panel: a word cloud that was constructed using the 500 most used keywords from the papers presented in the left panel.

meaningful information from images, as a promising toolbox to collect phenotypic information on a massive scale. The field has blossomed in recent years, producing a diverse array of computational tools to increase analytic efficiency, data dimensionality, and reproducibility. This technological advancement should be harnessed to produce more phenomic datasets, which will make our conclusions and inferences about biological phenomena more robust. We argue that $\mathrm{CV}$ is poised to become a basic component of the data analysis toolkit in ecology and evolution, enabling researchers to collect and explore phenomic-scale data with fewer systematic biases (e.g., from manual collection). Our review is intended to provide an entry point for ecologists and evolutionary biologists to the automatic and semi-automatic extraction of phenotypic data from digital images. We start with a general introduction to $\mathrm{CV}$ and its history, followed by some practical considerations for the choice of techniques based on the given data, and finish with a list of some examples and promising open-source CV tools that are suitable for the study of phenotypes.

\section{THE STRUCTURE OF DIGITAL IMAGES}

A two dimensional image is an intuitive way to record, store, and analyze organismal phenotypes. In the pre-photography era, ecologists and evolutionary biologists used drawings to capture the shapes and patterns of life, later to be replaced by analog photography, which allowed for qualitative assessment and simple, often only qualitative analysis of phenotypic variation. With the advent of digital photography, biologists could collect phenotypic data at unprecedented rates using camera stands, camera traps, microscopes, scanners, video cameras, or any other instrument with semiconductor image sensors (Goesele, 2004; Williams, 2017). Image sensors produce two-dimensional raster images (also known as bitmap images), which store incoming visible light or other electromagnetic signals into discrete, locatable picture elements - in short: pixels (Figure 2; Fossum and Hondongwa, 2014). Each pixel contains quantitative phenotypic information that is organized as an array of rows and columns, whose dimensions are also referred to as "pixel resolution" or just "resolution." An image with 1,000 rows and 1,500 columns has a resolution of $1,000 \times 1,500$ (=1,500,000 pixels, or 1.5 megapixels). The same applies for digital videos, which are simply a series of digital images displayed in succession, where the frame rate (measured as "frames per second" = fps) describes the speed of that succession.

On the pixel level, images or video frames can store variable amounts of information, depending on the bit depth, which refers to the number of distinct values that a pixel can represent (Figure 2). In binary images, pixels contain information as a single bit, which can take exactly two values - typically zero or one which represent black or white $\left(2^{1}\right.$ values $=2$ intensity values). Grayscale images from typical consumer cameras have a bit depth of eight, thus each pixel can take a value between 0 and 255 ( $2^{8}$ values $=256$ intensity values $)$, which usually represents a level of light intensity, also referred to as pixel intensity. Color images are typically composed of at least three sets of pixel arrays, also referred to as channels, each of which contain values for either red, green or blue (RGB; Figure 2). Each channel, when extracted from an RGB image, is a grayscale representation of the intensities for a single color channel. Through the 


\begin{tabular}{|c|c|}
\hline Bit depth & Number of values a pixel can take (e.g., 8 bit $=2 \wedge 8=256$ values) \\
\hline Computer vision & $\begin{array}{l}\text { Technical domain at the intersection of signal processing, machine learning, robotics and other scientific areas that is concerned with } \\
\text { the automated extraction of information from digital images and videos. }\end{array}$ \\
\hline Convolution & $\begin{array}{l}\text { Mathematical operation by which information contained in images are abstracted. Each convolutional layer produces a feature map, } \\
\text { which is passed on to the next layer. }\end{array}$ \\
\hline Deep learning & $\begin{array}{l}\text { Machine learning methods based on neural networks. supervised learning = algorithm learns input features from input-output pairs } \\
\text { (e.g., labeled images). unsupervised = algorithm looks for undetected patterns (e.g., images without labeling). }\end{array}$ \\
\hline Feature & $\begin{array}{l}\text { A measurable property or pattern. can be specific (e.g., edges, corners, points) or abstract (e.g., convolution via kernels), and } \\
\text { combined to vectors and matrices (feature maps). }\end{array}$ \\
\hline Feature detection & Methods for making pixel-level or pixel-neighborhood decisions on whether parts of an image are a feature or not. \\
\hline Foreground & $\begin{array}{l}\text { All pixels of interest in a given image, whereas the background constitutes all other pixels. the central step in computer vision is the } \\
\text { segmentation of all pixels into foreground and background. }\end{array}$ \\
\hline Hidden layer & $\begin{array}{l}\text { A connected processing step in neural networks during which information is received, processed (e.g., convolved), and passed on to } \\
\text { the next layer. }\end{array}$ \\
\hline Kernel & $\begin{array}{l}\text { A small mask or matrix to perform operations on images, for example, blurring, sharpening or edge detection. the kernel operation is } \\
\text { performed pixel wise, sliding across the entire image. }\end{array}$ \\
\hline Labeling & $\begin{array}{l}\text { Typically manual markup of areas of interest in an image by drawing bounding boxes or polygons around the contour. can be multiple } \\
\text { objects and multiple classes of objects per image. can also refer to assigning whole images to a class (e.g., relevant for species } \\
\text { identification). }\end{array}$ \\
\hline Machine learning & $\begin{array}{l}\text { Subset of artificial intelligence: the study and implementation of computer algorithms that improve automatically through experience. } \\
\text { (Mitchell, 1997). }\end{array}$ \\
\hline Measurement theory & $\begin{array}{l}\text { A conceptual framework that concerns the relationship between measurements and nature so that inferences from measurements } \\
\text { reflect the underlying reality intended to be represent (Houle et al., 2011). }\end{array}$ \\
\hline Neural network & $\begin{array}{l}\text { Deep learning algorithms that use multi layered ("deep") abstractions of information to extract higher level features from input via } \\
\text { convolution. }\end{array}$ \\
\hline Object detection & Methods for determining whether a pixel region constitutes an object that belongs to the foreground or not, based on its features. \\
\hline Pixel & $\begin{array}{l}\text { Short for picture element; the smallest accessible unit of a digital raster image. Pixels have finite values (= intensities), e.g., } 256 \text { in an } \\
\text { 8-bit grayscale image. }\end{array}$ \\
\hline Segmentation & $\begin{array}{l}\text { The classification of all pixels in an image into foreground and background, either manually by labeling the area of interest, or } \\
\text { automatically, by means of signal processing or machine learning algorithms. semantic segmentation = all pixels of a class, instance } \\
\text { segmentation = all instances of a class. }\end{array}$ \\
\hline Signal processing & $\begin{array}{l}\text { Technically correct: digital image processing (not to be confused with image analysis or image editing). subfield of engineering that is } \\
\text { concerned with the filtering or modification of digital images by means of algorithms and filter matrices (kernels), }\end{array}$ \\
\hline $\begin{array}{l}\text { Signal-to-noise } \\
\text { ratio (SNR) }\end{array}$ & $\begin{array}{l}\text { Describes the level of the pixels containing the desired signal (i.e., the phenotypic information) to all other pixels. Lab images typically } \\
\text { have a high SNR, field images a low SNR. }\end{array}$ \\
\hline Threshold algorithm & $\begin{array}{l}\text { Pixel-intensity based segmentation of images, e.g., based on individual pixel intensity (binary thresholding) or their intensity with } \\
\text { respect to their neighborhood (adaptive thresholding). Creates a binary mask which contains only black or white bixels. }\end{array}$ \\
\hline Training data & $\begin{array}{l}\text { Representative image dataset to train a machine learning algorithm. Can be created manually by labeling images, or semi-automatic } \\
\text { by using signal processing for segmentation. can contain single or multiple classes. }\end{array}$ \\
\hline Watershed algorithm & $\begin{array}{l}\text { The segmentation of images by treating the pixels as a topographic map of basins, where bright pixels have high elevation and dark } \\
\text { pixels have low elevation. }\end{array}$ \\
\hline
\end{tabular}

combination of pixel values at each location into triplets, colors are numerically represented. Today the industrial standard for color images is 24-bit depth, in which each color channel has a bit depth of eight and thereby can represent 256 colors. Thus, 24-bit RGB images can represent over 16 million color variations in each pixel $\left(2^{24}=256 \times 256 \times 256=16,777,216\right.$ intensity values), which already greatly surpasses the estimated 2.28 million of color variations that humans can perceive (Pointer and Attridge, 1998).

Today, high resolution image sensors are an affordable way to store externally visible phenotypic information, like color and shape. However, advanced image sensors can also combine information from different spectra other than the visible light, like infrared radiation, which can be used to quantify individual body temperatures. With thermal image sensors, biologists can estimate body surface temperatures, which are correlated with internal (core) body temperatures (Tattersall and Cadena, 2010), particularly in small animals like insects (Tsubaki et al., 2010; Svensson et al., 2020). Thermal imaging, or thermography, offers new opportunities for ecophysiological evolutionary research of how animals cope with heat or cold stress in their natural environments (Tattersall et al., 2009; Tattersall and Cadena, 2010; Svensson and Waller, 2013). Fluorescence spectroscopy is another way to quantify phenotypes in high throughput and with high detail. For example, plate readers typically used in microbial and plankton research, can combine light in the visible spectrum with images containing information of cell fluorescence or absorbance to an "image stack" (Roeder et al., 2012). Image stacks and the inclusion of multiple spectral channels provide a promising avenue of research toward capturing a more 

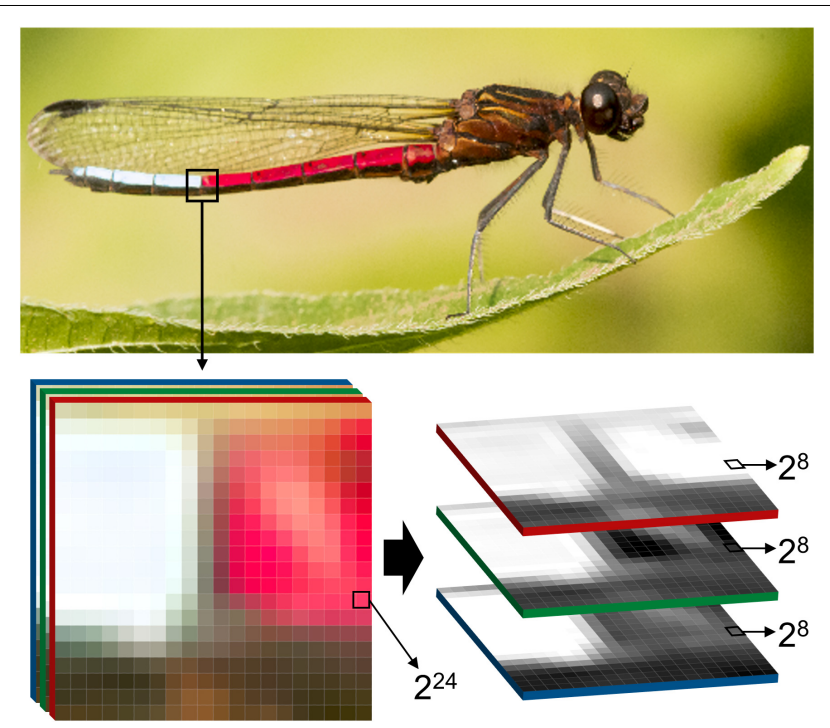

FIGURE 2 | The structure of digital images. Two-dimensional raster images, as produced by most commercially available cameras, are composed of three color channels red, green, blue $=\mathrm{RGB}$ ), each of which by itself is a grayscale image. The industrial standard for color representation on the pixel level is 24 bit ( $2^{24}=16777216$ possible color variations per pixel), which is achieved through additive mixing of each of the 8 bit channels $\left(2^{8}+2^{8}+2^{8}\right)$. This enormous range of color intensities among several million pixels is a potentially very high resolution representation of organismal traits, or the organism as a whole. Therefore, digital images are a useful medium for phenomics research, as they offer an inexpensive, memory efficient, and standardizable way to capture, store, and analyze complex phenotypes. The photograph shows a blue-tip jewel damselfly (Chlorocypha curta) in Cameroon (Africa, image by ES).

complete representation of the phenotype (Hense et al., 2008; Di et al., 2014).

\section{A BRIEF INTRODUCTION TO COMPUTER VISION}

CV-based extraction of phenotypic data from images can include a multitude of different processing steps that do not follow a general convention, but can be broadly categorized into preprocessing, segmentation, and measurement (Figure 3). These steps do not depict a linear workflow, but are often performed iteratively (e.g., preprocessing often needs to be adjusted according to segmentation outcomes) or in an integrated fashion (e.g., relevant data can already be extracted during preprocessing or segmentation).

\section{Preprocessing: Preparing an Image for Further Processing}

Independent of how much care has been taken during image acquisition, preprocessing is an important step to prepare images for the CV routines to follow. There is a wealth of image processing techniques that can be applied at this stage, such as transformations to reduce or increase noise (e.g., Gaussian blur) or enhance contrast (e.g., histogram adjustment).

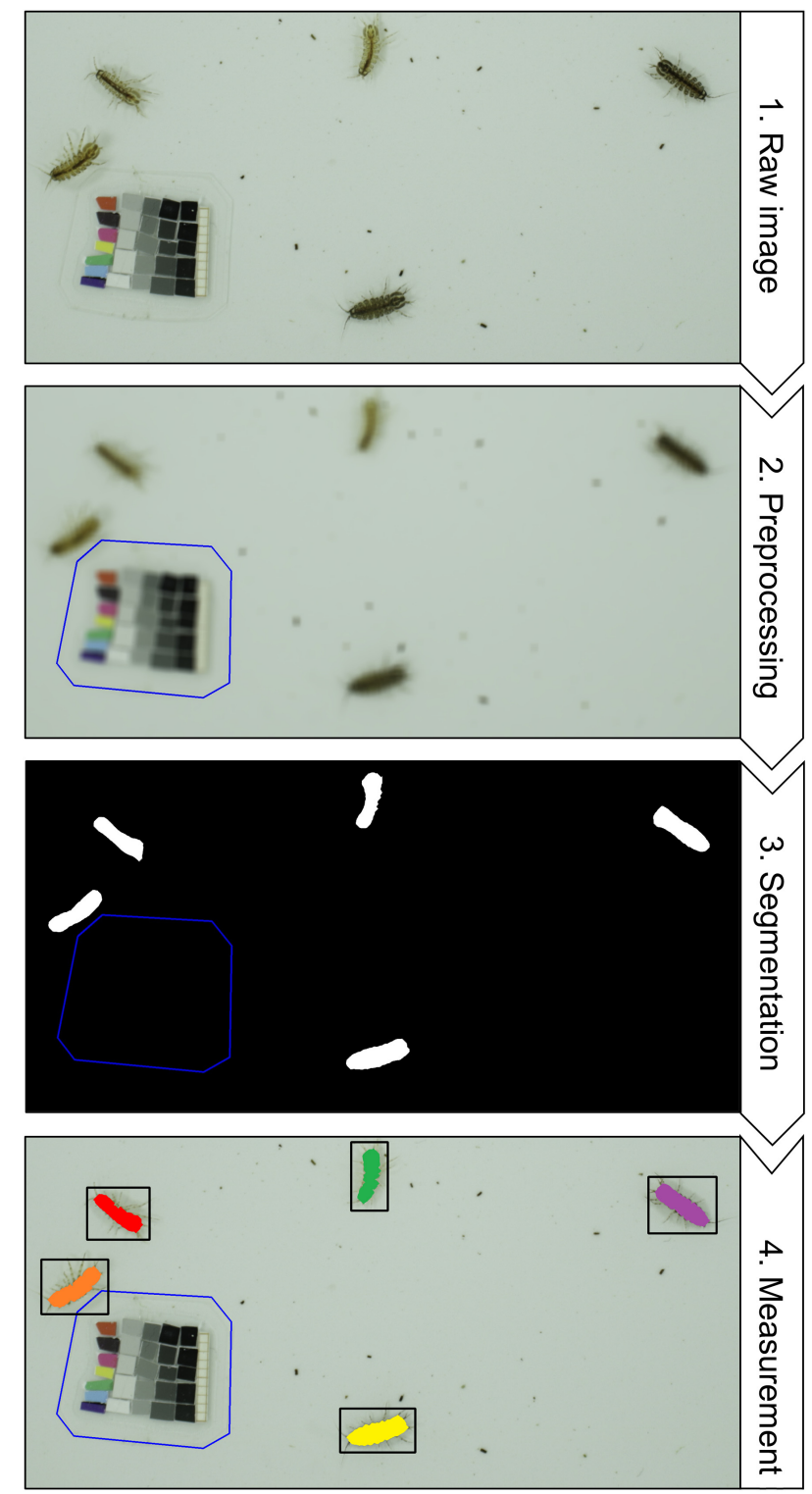

FIGURE 3 | A typical computer vision workflow using signal processing. (1) Raw image - The goal is to detect, count and measure freshwater isopods (Asellus aquaticus, image by MDL) from the raw image that was taken under controlled laboratory conditions. (2) Preprocessing - The operating principle of most signal processing workflows is that the objects of interest are made to contrast strongly from all other pixels, meaning that images should have a high signal-to-noise ratio (SNR. In this specific case a high SNR is already present, because the isopods are much darker than the tray they are sitting on and much larger than the fecal pellets and other detritus around them. To further increase the SNR, gaussian blur blends pixels in a given neighborhood (=kernel size), which effectively removes the smaller dark objects. The reference card gets excluded manually, and can be used to convert pixels to millimeters and to correct the color space. (3) Segmentation - Using a thresholding algorithm all connected pixels that are above a specific grayscale value and larger than a specified area are designated foreground (white) and all pixels become background (black). The output from this step is referred to as a "binary mask". (4) Measurement - Now the white pixels from the binary mask can be used to locate the areas of interest in the raw image and to extract information from them. Discrimination between multiple instances of the same class is referred to as instance segmentation. 
Images can also be masked or labeled as a way to filter the image so that subsequent steps are applied to the intended portions of each image. Defining the appropriate coordinate space (i.e., pixel-to-mm ratios) is also part of preprocessing. Finally, certain machine learning techniques such as deep learning require an enormous amount of data, which may require data augmentation: the addition of slightly modified copies of existing data or the addition of newly created synthetic data (Shorten and Khoshgoftaar, 2019). Overall, preprocessing tasks are highly specific to the respective study system, image dataset or $\mathrm{CV}$ technique, and may initially require some fine-tuning by the scientist to ensure data quality, which, however, can typically be automated afterward.

\section{Segmentation: Separation of "Foreground" from "Background"}

The central step in any phenotyping or phenomics related CV pipelines is the segmentation of images into pixels that contain the desired trait or character (foreground) and all other pixels (background). In its most basic form, segmentation of grayscale images can be done by simple signal processing algorithms, such as threshold (Zhang and $\mathrm{Wu}, 2011$ ) or watershed (Beucher, 1979). Similarly, feature detection algorithms examine pixels and their adjacent region for specific characteristics or key points, e.g., whether groups of pixel form edges, corners, ridges or blobs (Rosten and Drummond, 2006). Videos or multiple images of the same scene provide an additional opportunity for segmentation: foreground detection can detect changes in image sequences to determine the pixels of interest (e.g., a specimen placed in an arena, or animals moving against a static background), while subsequent background subtraction isolates the foreground for further processing (Piccardi, 2004). Finally, object detection describes the high level task of finding objects (organisms, organs, structures, etc.) in an image, which is typically addressed through classical machine learning or deep learning (see section "A History of Computer Vision Methods”; LeCun et al., 2015; Heaton, 2020; O'Mahony et al., 2020). In classical machine learning, features have to be first engineered or extracted from a training dataset using feature detectors, then used to train a classifier, and finally applied to the actual dataset (Mitchell, 1997). Deep learning algorithms are a family of machine learning methods based on artificial neural networks that "learn" what constitutes the object of interest during the training phase (LeCun et al., 2015; Heaton, 2020). With sufficient training using labeled images (and in some cases unlabeled images - see Box 2), deep learningpowered object detection algorithms can be highly accurate and often greatly outperform pre-existing object recognition methods (Krizhevsky et al., 2012; Alom et al., 2018) - in some cases even human experts, for example, when identifying species (BuettiDinh et al., 2019; Valan et al., 2019; Schneider et al., 2020b). Each of these approaches has advantages and limitations, which mostly depend on the noise level within the images, the size of the dataset, and the availability of computational resources (see section "Practical Considerations for Computer Vision" and Figure 4).

\section{Measurement: Extraction of Phenotypic Data}

Computer Vision can retrieve a multitude of phenotypic traits from digital images in a systematic and repeatable fashion (see Table 1). In the simplest case, CV may measure traits that are established in a given study system, such as body size (e.g., length or diameter) or color (e.g., brown phenotype vs. blue phenotype). In such cases, switching from a manual approach to a semi- or fully automatic CV approach is straightforward, because the target traits are well embedded in existing statistical and conceptual frameworks. The main benefits from CV are that costly manual labor is reduced and that the obtained data becomes more reproducible, because the applied CV analysis pipeline can be stored and re-executed. However, just as manual measurements require skilled personnel to collect high quality data, great care needs to be taken when taking images so that their analysis can provide meaningful results (also see section "Image Quality: Collect Images That Are Maximally Useful”). It is also possible to increase the number of dimensions without much extra effort and without discarding the traditionally measured traits (Table 1). For example, in addition to body size, one could extract body shape traits, i.e., the outline of the body itself (i.e., contour coordinates of the foreground), and texture (i.e., all pixel intensities within the foreground). Such high dimensional traits can be directly analyzed using multivariate statistics, or transformed into continuous low dimensional traits, such as continuous shape features (circularity or area), texture features (color intensity or variation, pixel distribution), or moments of the raw data (Table 1).

\section{A HISTORY OF COMPUTER VISION METHODS}

$\mathrm{CV}$ is an interdisciplinary field at the intersection of signal processing and machine learning (Figure 4; Mitchell, 1997), which is concerned with the automatic and semiautomatic extraction of information from digital images (Shapiro and Stockman, 2001). The field is now close to celebrating its 6th decade. It first emerged in the late 1950s and early 1960s, in the context of artificial intelligence research (Rosenblatt, 1958). At the time, it was widely considered a stepping-stone in our search for understanding human intelligence (Minsky, 1961). Given its long history, a wide-variety of $\mathrm{CV}$ techniques have emerged since its inception, but they all contain variations of the same basic mechanism. CV is, from the methodological standpoint, the process of extracting meaningful features from image data and then the use of such features to perform tasks, which, as described above, may include classification, segmentation, recognition, and detection, among others. In this section, we will not aim at presenting an all-encompassing review of all CV methods, but rather to identify the major trends in the field and highlight the techniques that have proved useful in the context of biological research. It is worth noting that even classical CV approaches are still routinely used in the modern literature, either in isolation or, most commonly, in combination with others. In a large 
BOX 2 | An overview of the main deep learning architectures and approaches.

\section{Families of network topologies}

A. Deep convolutional network - A large and common family of neural networks composed an input layer, an output layer and multiple hidden layers. These networks feature convolution kernels that process input data and pooling layers that simplify the information processed through the convolutional kernels. For certain tasks, the input can be a window of the image, rather than the entire image.

B. Deconvolutional Network - A smaller family of neural networks that perform the reverse process when compared to convolutional networks. It starts with the processed data (i.e., the output of the convolutional network) and it aims to separate what has been convoluted. Essentially, it constructs upward from processed data (e.g., reconstructs an image from a label).

C. Generative Adversarial Network - A large family of networks composed of two separate networks, a generator and a discriminator. The generator is trained to generate realistic data, while the discriminator is trained to differentiate between generated data from actual samples. Essentially, in this approach, the objective is for the generator to generate such realistic data that the discriminator cannot tell it apart from samples.

D. Autoencoders - A family of networks is trained in an unsupervised manner. The autoencoder aims to learn how to robustly represent the original dataset, oftentimes in smaller dimensions, even in the presence of noise. Autoencoders are composed of multiple layers, and it can be divided into two main parts: the encoder and the decoder. The encoder maps the input into the representation and the decoder uses the representation to reconstruct the original input.

E. Deep Belief Network - A family of generative networks that are composed of multiple layers of hidden units, in which there can be connections between layers but not within units within layers. Deep belief networks can be conceived as being composed of multiple simpler networks, where each subnetwork's hidden layer acts as a visible layer to another subnetwork.

\section{Learning Classes}

A. Supervised Learning - Training data is provided when fitting the model. The training dataset is composed of inputs and expected outputs. Models are tested by making predictions based on inputs and comparing them with expected outputs.

B. Unsupervised Learning - No training data is provided to the model. Unsupervised learning relies exclusively on inputs. Models trained using unsupervised learning are used to describe or extract relationships in image data, such as clustering or dimensionality reduction.

C. Reinforcement Learning - The learning process occurs in a supervised manner, but not through the use of static training datasets. Rather, in reinforcement learning, the model is directed toward a goal, with a limited set of actions it may perform, and model improvement is obtained through feedback. The learning itself occurs exclusively through feedback obtained based on past action. This feedback can be quite noisy and delayed.

D. Hybrid Learning Problems

Semi-Supervised Learning - Semi supervised learning relies on training datasets where only a small percentage of the training dataset is labeled, with the remaining images having no label. It is a hybrid in between supervised and unsupervised learning, since the model has to make effective use of unlabeled data while relying only partially on labeled ones.

Self-Supervised Learning - Self supervised learning uses a combination of unsupervised and supervised learning. In this approach, supervised learning is used to solve a pretext task for which training data is available (or can be artificially provided), and whose representation can be used to solve an unsupervised learning problem. Generative adversarial networks rely on this technique to learn how to artificially generate image data.

\section{Other learning Techniques}

A. Active Learning - During active learning, the model can query the user during the learning process to require labels for new data points. It requires human interaction and it aims to being more efficient about what training data is used by the model.

B. Online Learning - Online learning techniques are often used in situations where observations are streamed through time and in which the probability distribution of the data might drift over time. In this technique, the model is updated as more data becomes available, allowing the model itself to change through time.

C. Transfer Learning - Transfer learning is a useful technique when training a model for a task that is related to another task for which a robust model is already available. Essentially, it treats the already robust model as a starting point from which to train a new model. It greatly diminishes the training data needs of supervised models and it is, therefore, used when the available training data is limited.

D. Ensemble Learning - As mentioned in the main text, ensemble learning refers to a learning technique in which multiple models are trained either in parallel or sequentially and the final prediction is the result of the combination of the predictions generated by each component.

part, methodological choices in CV are highly domain-specific (see section "Practical Considerations for Computer Vision" and Figure 4).

\section{First Wave - Hand-Crafted Features}

The first wave of $\mathrm{CV}$ algorithms is also the closest one to the essence of $\mathrm{CV}$, namely, the process of extracting features from images. Starting with the work of Larry Roberts, which aimed at deriving 3D information from 2D images (Roberts, 1963), researchers in the 1970s and 1980s developed different ways to perform feature extraction from raw pixel data. Such features tended to be low-level features, such as lines, edges, texture or lighting, but provided us with the initial basic geometric understanding of the data contained in images. A notable example of such algorithms is the watershed algorithm. First developed in 1979 (Beucher, 1979), the watershed algorithm became popular in biological applications in the 1990s, being initially used to quantify elements and extract morphological measurements from microscopic images [e.g., (Bertin et al., 1992; Rodenacker et al., 2000)]. This algorithm treats images as a topographic map, in which pixel intensity represents its height, and attempts to segment the image into multiple separate "drainage basins." Certain implementations of the watershed algorithm are still routinely used in signal processing (Figure 4), and can be effectively used to process biological images such as those obtained through animal or plant cell microscopy (McQuin et al., 2018). Other initial lowlevel hand-crafted approaches that achieved popularity include 


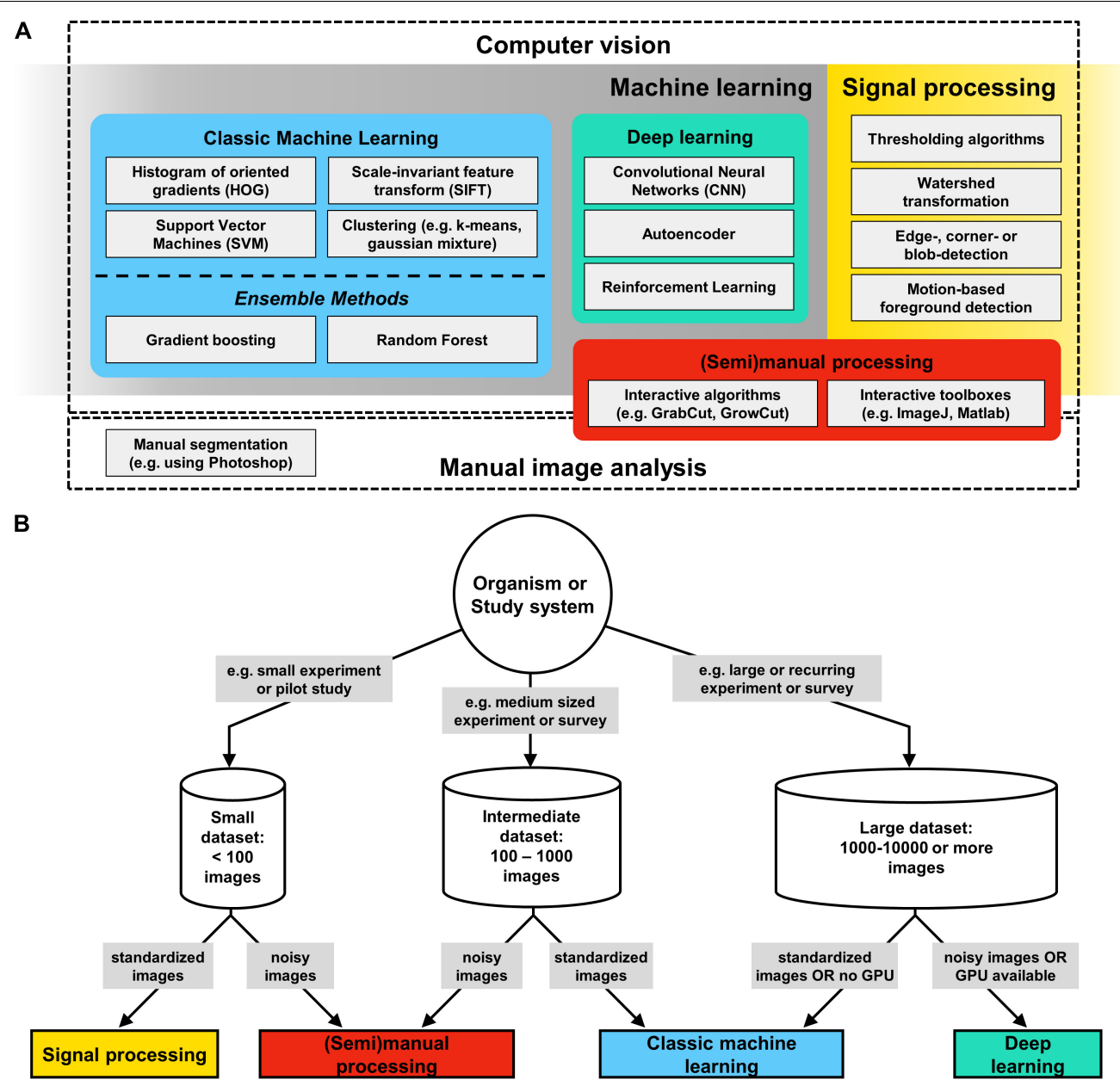

FIGURE 4 | Computer vision (CV) methods overview. (A) CV is a field at the intersection of machine learning and signal processing which is concerned with the automatic and semiautomatic extraction of information from digital images. (B) Decision tree for CV methods: begin by considering the size of a given image dataset, whether it is complete, e.g., after an experiment, or whether there will be continued future additions, e.g., as part of a long term experiment or field survey. The next assessment should be about the signal-to-noise ratio (SNR) in your images: images taken in the laboratory typically have a high degree of standardization and thus a very high SNR, which makes them suitable for a signal processing approach. In contrast to signal processing, where segmentation results are immediately available, all machine learning image analysis pipelines include iterative training and validation phases, followed by a final testing phase. Such a workflow can be complex to initiate, but pays off in the long run by providing segmentation results that become increasingly robust if more training data is supplied over time. Deep learning algorithms require large training datasets (several 1,000-10,000s) and a powerful graphics processing unit (GPU), but are less sensitive to noise and idiosyncrasies of the foreground. Thus, for large and continuously growing data sets, or for recurring image analysis tasks, deep learning has become the standard approach for segmentation.

the Canny and Sobel filters [edge detection (Canny, 1986; Kanopoulos et al., 1988)] and Hough transforms [ridge detection (Duda and Hart, 1972)].

Another approach that gained popularity in the CV literature in the early 1990s was principal component analysis (PCA). In a PCA, independent, aggregate statistical features are extracted from multidimensional datasets. These can be used, for example, in classification. One of the most notable uses of PCA in the context of CV was the eigenfaces approach (Turk and Pentland, 1991). Essentially, Turk and Pentland (1991) noted that one could decompose a database of face images into eigenvectors (or characteristic images) through PCA. These eigenvectors could then be linearly combined to reconstruct any image in the original dataset. A new face could be decomposed into statistical features and further compared to other known images in a multidimensional space. Similar pioneering approaches emerged in the context of remote sensing research, in which spectral image data was decomposed into its eigenvectors (Bateson and Curtiss, 1996; Wessman et al., 1997). PCA has notably found many other uses in biology [e.g., (Ringnér, 2008)].

In the late 1990s and early 2000s, Scale Invariant Feature Transform [SIFT (Lowe, 1999, 2004)] and Histogram of Oriented Gradients [HOG (Dalal and Triggs, 2005)] were developed. Both SIFT and HOG represent intermediate-level local features that can be used to identify keypoints that are shared across images. In both approaches, the first step is the extraction of these intermediate-level features from image data, followed by a feature 
TABLE 1 | Classes of phenotypic data.

\begin{tabular}{|c|c|c|}
\hline Trait type & Low dimensional & High dimensional \\
\hline $\begin{array}{l}\text { Specific / directly } \\
\text { measurable }\end{array}$ & $\begin{array}{l}\text { Size, discrete color ("red phenotype" vs. "blue phenotype"), and morphotype scoring (e.g., } \\
\text { benthic vs. limnetic) }\end{array}$ & $\begin{array}{l}\text { Shape coordinates, texture maps, and } \\
\text { landmarks }\end{array}$ \\
\hline Abstract / derived & $\begin{array}{l}\text { Shape (e.g., circularity, area) and texture features (e.g., mean, SD, uniformity), moments, } \\
\text { principal components, and hypervolumes }\end{array}$ & Matrices and activation maps \\
\hline
\end{tabular}

matching step that tries to identify those features in multiple images. Finding keypoints across images is an essential step in many CV applications in biology, such as object detection, landmarking (Houle et al., 2003), and image registration (Mäkelä et al., 2002). These intermediate-level features have several advantages over the lower-level features mentioned above, most notably the ability to be detected in a wide-variety of scales, noise, and illumination. Another key aspect of SIFT and HOG features is that they are generally invariant to certain geometric transformations, such as uniform scaling and simple affine distortions.

\section{Second Wave - Initial Machine-Learning Approaches}

While the use of hand-crafted features spurred much of the initial work in $\mathrm{CV}$, soon it became apparent that without image standardization, those low- and intermediate-level features will often fall short of producing sufficiently robust CV algorithms. For example, images belonging to the same class can often look very different and the identification of a common set of shared low-level features can prove to be quite challenging. Consider, for instance, the task of finding and classifying animals in images: two dog breeds can look quite different, despite belonging to the dog class (e.g., Chihuahua vs. Bernese mountain $\operatorname{dog}$ ). As such, while the initial feature-engineering approaches were essential for the development of the field, it was only with the advent of machine-learning that $\mathrm{CV}$ acquired more generalizable applications.

Machine learning algorithms for $\mathrm{CV}$ can be divided in two main categories (but see Box 2): supervised and unsupervised (Hinton and Sejnowski, 1999). Unsupervised algorithms attempt to identify previously unidentified patterns on unlabeled data. In other words, no supervision is applied to the algorithm during learning. While it can be argued that PCA was one of the first successful unsupervised learning algorithms applied directly to $\mathrm{CV}$, here we group PCA with "first wave" tools due to its use as a feature extractor. Other unsupervised learning algorithms commonly used in CV include clustering techniques, such as k-means (Lloyd, 1982) and gaussian mixture models [GMM (Reynolds and Rose, 1995)]. Clustering algorithms represented some of the first machine learning approaches for CV. Their aim is to find an optimal set of objects (or components) that are more similar to each other than to those in other sets. This type of approach allowed researchers to find hidden patterns embedded in multidimensional data, proving useful for classification and segmentation tasks. For example, GMM has been extensively used to classify habitat using satellite image data (Zhou and Wang, 2006), to segment MR brain images (Greenspan et al., 2006), and classification of animals from video (Edgington et al., 2006), to name a few.

However, it is in the supervised domain that machine learning for CV has been most successful (Heileman and Myler, 1989). In supervised learning approaches, the user supplies labeled training data in the form of input-output pairs (Box 2). The ML algorithm iteratively "learns" a function that maps input into output for the labeled training data. Among the initial supervised approaches for CV, Support Vector Machines (SVM) were by far the most common approach (Cortes and Vapnik, 1995). Given a certain image dataset and their corresponding labels (e.g., classes in a classification task), SVMs find the feature space that maximizes the separation between the classes of interest (referred to as hyperplane). An essential aspect of SVMs is that such learned decision boundaries separating the classes can be non-linear in the original feature space, allowing the model to separate classes that would not be separable by a purely linear technique (Cortes and Vapnik, 1995). Support vector machines have been widely used in ecological research, e.g., for image classification (SanchezHernandez et al., 2007) and image recognition (Hu and Davis, 2005), among others.

\section{Third Wave - Ensemble Methods}

While SVMs were extremely successful in CV and spurred much of the supervised work that happened afterward, it became clear by the early 2000s that single estimators often underperformed approaches combining the predictions of several independent estimators, an approach known as ensemble methods (Sollich and Krogh, 1996; Dietterich, 2000). Ensemble methods represent a slightly different philosophical approach to machine learning, in which multiple models are trained to solve the same task and their individual results are combined to obtain an even better model performance. Several ensemble methods have been developed in the literature, but they are generally divided in two main families: bagging and boosting.

Bagging approaches combine several models that were trained in parallel through an averaging process (Bauer and Kohavi, 1999). Each underlying model is trained independently of the others based on a bootstrap resample of the original dataset. As a consequence, each model is trained with slightly different and (almost) independent data, greatly reducing the variance in 
the combined model predictions. A classical example of bagging approach is the random forest algorithm (Breiman, 2001), in which multiple learning trees are fitted to bootstrap resamples of the data and posteriorly combined through mean averaging (or majority vote). In biology, bagging approaches have been used for environmental monitoring (Mortensen et al., 2007), sample identification (Lytle et al., 2010), among others. Boosting, on the other hand, combines learners sequentially rather than in parallel (Bauer and Kohavi, 1999). Among boosting algorithms, gradient boosting (Friedman, 2000) is one of the most widely used in CV. In gradient boosting, models are combined in a cascade fashion, such that a downstream model is fitted to the residuals of upstream models. As a consequence, while each individual model in the cascade is only weakly related to the overall task, the combined algorithm (i.e., the entire cascade) represents a strong learner that is directly related to the task of interest (Friedman, 2000). Since this approach, if unchecked, will lead the final model to overfit the training data, regularization procedures are usually applied when using gradient boosting.

\section{Fourth Wave - Deep Learning}

Deep learning approaches are, at the time of this writing, the state-of-the-art in $\mathrm{CV}$ and have recently become more accessible through the community-wide adoption of codesharing practices. Deep learning refers to a family of machine learning methods based on hierarchical artificial neural networks, most notably, convolutional neural networks (CNN). Networks with dozens or hundreds of hidden layers (i.e., deep neural networks) allow for the extraction of high-level features from raw image data (LeCun et al., 2015). While they have only recently become widespread, the history of artificial neural networks is at least as old as the field of CV itself. One of first successful attempts in the study of artificial neural networks was the "perceptron" (Rosenblatt, 1958), a computer whose hardware design was inspired by neurons, and which was used to classify a set of inputs into two categories. This early work, while successful, was largely restricted to linear functions and therefore could not deal with non-linearity, such as exclusive-or (XOR) functions (Minsky and Papert, 1969). As a consequence, artificial neural network research remained rather understudied until the early 1980s when training procedures for multi-layer perceptrons were introduced (i.e., backpropagation; Rumelhart and McClelland, 1987). Even then, multi-layer approaches were computationally taxing and the hardware requirements represented an important bottleneck to research in neural network based $\mathrm{CV}$, which remained disfavored compared to much lighter approaches, such as SVMs.

When compared to the hand-crafted features that dominated the field for most of its history, neural networks learn features from the data itself, therefore eliminating the need for feature engineering (LeCun et al., 2015). In a large part, deep learning approaches for $\mathrm{CV}$ have only emerged in force due to two major developments at the beginning of the 21st century. On one side, hardware capability greatly increased due to high consumer demand for personal computing and gaming. On the other, there was a widespread adoption of the internet, leading to an exponential increase in data availability through shared image databases and labeled data. Today, deep learning is a general term that encompasses a wide-variety of approaches that share an architectural commonality of relying on training neural networks with multiple hidden layers (LeCun et al., 2015; O'Mahony et al., 2020). However, this superficial similarity hides a considerable array of differences between different algorithms and one could say that the field of deep learning is as diverse as the domains in which CV is applied. In ecology and evolution, deep neural networks have been used for essentially any CV task, many of each can be seen in other parts of this review. We present some of the most relevant classes of deep learning approaches in Box 2.

\section{PRACTICAL CONSIDERATIONS FOR COMPUTER VISION}

\section{Before Taking Images}

Measurement Theory: Define Your Traits Thoughtfully

Defining meaningful phenotypes is deceptively challenging. Traditionally, biologists relied on intuition and natural history conventions to define phenotypes without quantitative verifications of their relevance for biological questions. When deciding what to measure, we suggest that researchers consider measurement theory, a qualitative formalization of the relationship between actual measurements and the entity that the measurements are intended to represent (Houle et al., 2011). In phenomics using $\mathrm{CV}$, we recommend that researchers adhere to the following three principles: i) Ensure that the measurements are meaningful in the theoretical context of research questions. ii) Remember that all measurements are estimates. Measurements without uncertainties should always be avoided. iii) Be careful with units and scale types, particularly when composite values, such as the proportion of one measurement over another, are used as a measurement. Wolman (2006) and Houle et al. (2011) give details of measurement theory and practical guidelines for its use in ecology and evolutionary biology.

\section{Image Quality: Collect Images That Are Maximally Useful}

As a general rule of thumb, images taken for any $\mathrm{CV}$ analysis should have a signal-to-noise ratio (SNR) sufficiently high so that the signal (i.e., the phenotypic information) is detectable from the image background. High SNR can be achieved by using high resolution imaging devices (e.g., DSLR cameras or flatbed scanners), ensuring that the object is in focus and always maintains the same distance to the camera (e.g., by fixing the distance between camera and object), and by creating a high contrast between object and background (e.g., by using backgrounds that are of contrasting color or brightness to the organism or object). We recommend to iteratively assess suitability of imaging data early on in a project and adjust if necessary. This means taking pilot datasets, processing them, measuring traits, estimating measurement errors, and then updating the image collection process. Moreover, it is good practice to include a color or size reference whenever possible 
(e.g. see Figure 3). It helps researchers to assess if the image has sufficient SNR, increases reproducibility, and helps to evaluate measurement bias, as we discuss in the next section.

\section{On Measurement Error}

Because conventional phenotyping methods are often timeconsuming and depend on what is possible within a given period of time, biologists are rarely able to evaluate measurement errors and deal with them in downstream analyses. A major advantage of $\mathrm{CV}$ lies in its ability to assess the (in)accuracy of measurements easily. Formally, measurement inaccuracy is composed of imprecision and bias, corresponding to random and systematic differences between measured and true values, and can be expressed as the following relationship:

$$
\text { inaccuracy }=\text { imprecision }+ \text { bias }^{2}
$$

(Grabowski and Porto, 2017; Tsuboi et al., 2020). These two sources of errors characterize distinct aspects of a measurement: precise measurements may still be inaccurate if biased, and unbiased measurements may still be inaccurate if imprecise (Figure 5). Measurement imprecision can be evaluated by the coefficient of variation (standard deviation divided by the mean) of repeated measurements. Bias requires a knowledge of true values.

We ultimately need to understand if a measurement is sufficiently accurate to address the research question at hand. Repeatability is a widely used estimator of measurement accuracy in ecology and evolutionary biology (Wolak et al., 2012), which in our notation could be expressed as

$$
\text { repeatability }=1-\frac{\text { inaccuracy }}{\text { total variance }}
$$

This expression clarifies that the repeatability depends both on measurement inaccuracy and total variance in the data. For example, volume estimates of deer antlers from $3 \mathrm{D}$ photogrammetry have an average inaccuracy of $8.5 \%$, which results in repeatabilities of $67.8-99.7 \%$ depending on the variance in antler volume that a dataset contains (Tsuboi et al., 2020). In other words, a dataset with little variation requires more accurate measurement to achieve the same repeatability as a dataset with more variation. Therefore, the impact of measurement error has to be evaluated in the specific context of data analysis.

One way to improve measurement precision is to repeat a measurement and take their mean as the representative measurement. For example, when measuring deer antler volume estimated from 3D photogrammetry (Figure 6E; Tsuboi et al., 2020 ), it was found that $70 \%$ of the total inaccuracy arose from the error in scaling arbitrary voxel units into real volumetric units. Therefore, by using the mean of two estimates obtained from two copies of an image that are scaled twice independently, the inaccuracy dropped to $5.5 \%$. However, the opportunity to improve accuracy by repeated measurements is limited if a majority of error arises from the stored images themselves. For this reason, we recommend always taking repeated images of the same subject at least for a subset of data. This will allow evaluating the magnitude of error due to images relative to the error due to

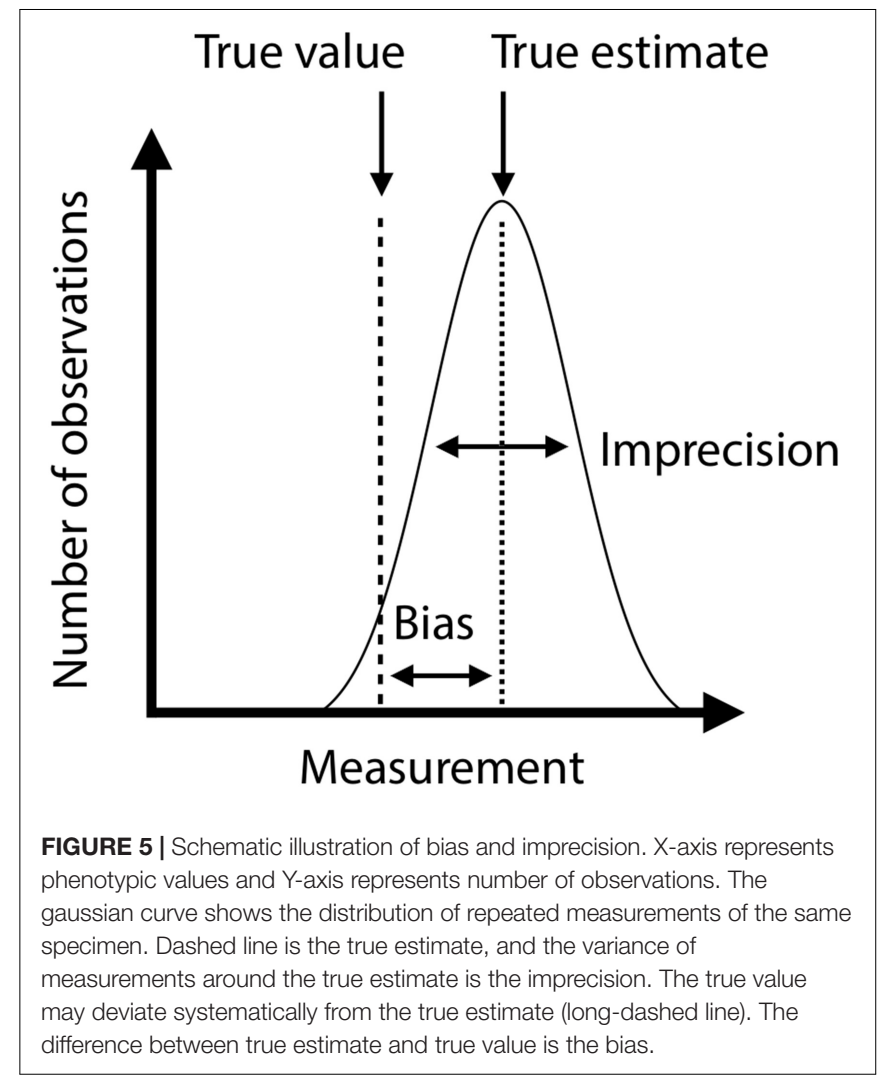

acquisition of measurements from images. If the error caused by images is large compared to the error caused by data acquisition, it may be necessary to modify imaging and/or preprocessing protocol to increase SNR.

Assessing measurement bias requires separate treatments. When linear (length) or chromatic (color) measurements are obtained from images, it is good practice to include size and color scales inside images to estimate bias as the difference between known pixel intensities in the image and the included scale (i.e., the reference card in Figure 3). Knowing the true value may be difficult in some cases, such as area or circularity (Figure 6C; Hoffmann et al., 2018), since they are hard to characterize without $\mathrm{CV}$. When multiple independent methods to measure the same character exist, we recommend using them on sample data to determine the bias of one method relative to the other.

\section{After Taking Images Selecting a CV Pipeline: As Simple as Possible, as Complex as Necessary}

When using CV tools there are usually many different ways to collect a specific type of phenotypic information from images (Figure 4). Therefore, one of the first hurdles to overcome when considering the use of $\mathrm{CV}$ is selecting the appropriate technique from among a large and growing set of choices. The continued emergence of novel algorithms to collect, process and analyze image-derived data may sometimes make us believe that any "older" technology is immediately outdated. Deep learning, specifically CNNs, is a prominent example of an innovation in 

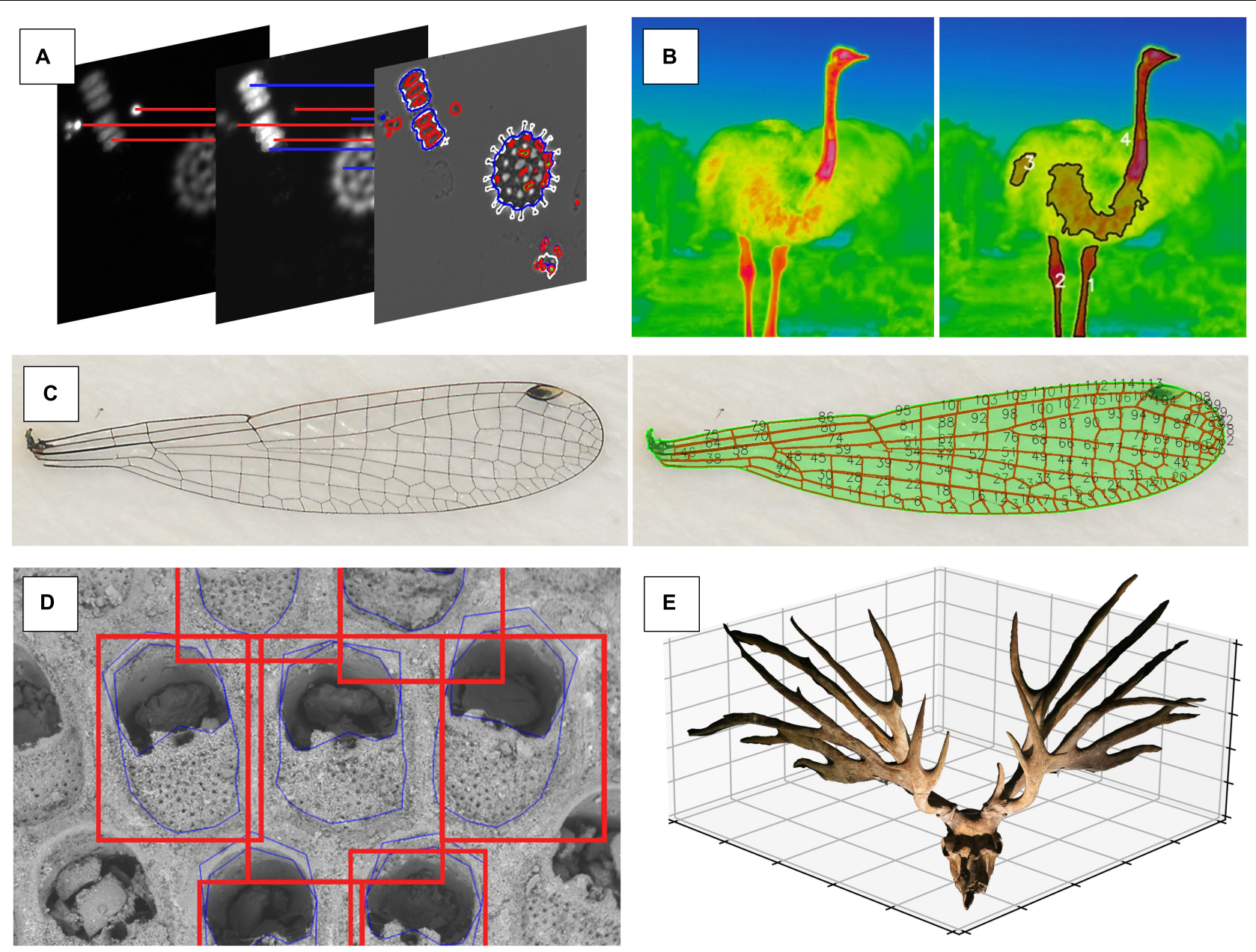

FIGURE 6 | Different types of high dimensional phenotypic data that are collected using a fully or semi-automatic computer vision approach. (A) Morphology and fluorescence traits of phytoplankton communities are represented through a combination of shape features (e.g., circularity, perimeter length, and area) and texture features (e.g., blob intensity and distribution within the cell) from images showing fluorescence intensity (images by Irene Gallego). (B) In ostriches (Struthio camelus), surface temperatures of bare body parts without feathers (necks and legs) are detected using signal processing (image by ES). (C) Signal processing approach that captures individual domains of a damselfly wing via thresholding (image by MT). (D) Ensemble-based approach to shape prediction of individual zooids within a bryozoan colony (image by AP). (E) 3D image of the skull of extinct deer Eucladoceros dicranios from which we can measure linear, area, and volumetric measurements of antler features (image by MT).

$\mathrm{CV}$ that was frequently communicated as so "revolutionary" and "transformative" that many scientists believed it would replace all existing methods. However, despite the success of CNNs, there are many cases where they are inappropriate or unfeasible, e.g., due to small sample sizes, hardware or time constraints, or because of the complexity that deep learning implementations entail, despite many efforts to make this technology more tractable (see Table 2). We discourage readers from defaulting to using the newest technology stacks; rather, we suggest that researchers be pragmatic as to which is the fastest and simplest way to get the phenotypic information of desire from any given set of images.

Begin by considering the size of a given image dataset, whether it is complete, or whether there will be continued data additions, e.g., as part of a long term experiment or field survey. As a rough rule of thumb, if a dataset encompasses only a thousand images or fewer, consider it "small"; if a dataset has thousands to tens of thousands images, consider it "large" (see Figure $\mathbf{4}$ for methodological suggestions for each case). The next assessment should be about the SNR in your images: images taken in the laboratory typically have a high degree of standardization, e.g., controlled light environment or background, and thus a very high SNR. Field images can also have a high SNR, for example, if they are taken against the sky or if the trait of question is very distinct from the background through bright coloration. If the dataset is "small" and/or has high SNR, it may not be necessary to use the more sophisticated CV tools; instead, signal processing, e.g., threshold or watershed algorithms, may already be sufficient for segmentation although typically some pre- and post-processing is typically still required (e.g., blurring to remove noise, "morphology"-operations to close gaps, or masking false positives). 
TABLE 2 | Select examples of recent open source computer vision libraries with a biology-context.

\begin{tabular}{|c|c|c|c|c|c|c|c|}
\hline Year & Name & References & Repository & Purpose & Application type & Description & Techniques \\
\hline 2021 & Phenopype & Lürig, 2021 & https://github.com/mluerig/phenopype & $\begin{array}{l}\text { Object detection, } \\
\text { feature extraction, and } \\
\text { motion tracking }\end{array}$ & Python package & $\begin{array}{l}\text { Multi-purpose High } \\
\text { throughput } \\
\text { phenotyping }\end{array}$ & Signal processing \\
\hline 2020 & EB-Net & Le et al., 2020 & $\begin{array}{l}\text { https://github.com/linhlevandlu/CNN_Beetles } \\
\text { _Landmarks }\end{array}$ & $\begin{array}{l}\text { Keypoint and feature } \\
\text { detection }\end{array}$ & Python application & Insect morphometrics & Deep learning \\
\hline 2020 & ML-morph & $\begin{array}{l}\text { Porto and Voje, } \\
2020\end{array}$ & https://github.com/agporto/ml-morph & $\begin{array}{l}\text { Landmark detection; } \\
\text { geometric } \\
\text { morphometrics }\end{array}$ & Python package & $\begin{array}{l}\text { High throughput } \\
\text { morphometrics }\end{array}$ & $\begin{array}{l}\text { Classic machine } \\
\text { learning, ensemble } \\
\text { Methods }\end{array}$ \\
\hline 2018 & AutoMorph & $\begin{array}{l}\text { Hsiang et al., } \\
2018\end{array}$ & https://github.com/HullLab/AutoMorph & $\begin{array}{l}\text { Object detection and } \\
\text { feature extraction }\end{array}$ & Python package & $\begin{array}{l}\text { High throughput } \\
\text { segmentation }\end{array}$ & Signal processing \\
\hline 2018 & DeepMerkat & $\begin{array}{l}\text { Weinstein, } \\
2015\end{array}$ & https://github.com/bw4sz/DeepMeerkat & $\begin{array}{l}\text { Object detection, } \\
\text { classification }\end{array}$ & Python application & $\begin{array}{l}\text { Background } \\
\text { subtraction and image } \\
\text { classification for } \\
\text { stationary cameras in } \\
\text { ecological videos }\end{array}$ & $\begin{array}{l}\text { Signal processing, } \\
\text { deep learning }\end{array}$ \\
\hline 2018 & WorMachine & $\begin{array}{l}\text { Hakim et al., } \\
2018\end{array}$ & https://github.com/adamhak/WorMachineClient & $\begin{array}{l}\text { Object detection and } \\
\text { feature extraction }\end{array}$ & Matlab application & $\begin{array}{l}\text { Integrated image } \\
\text { processing and feature } \\
\text { extraction }\end{array}$ & $\begin{array}{l}\text { Signal processing, } \\
\text { classic machine } \\
\text { learning; deep learning }\end{array}$ \\
\hline 2017 & ClickPoints & $\begin{array}{l}\text { Gerum et al., } \\
2017\end{array}$ & https://github.com/fabrylab/clickpoints & $\begin{array}{l}\text { Labeling, label } \\
\text { evaluative }\end{array}$ & Python package & Interactive labeling tool & Signal processing \\
\hline 2017 & PlantCV & $\begin{array}{l}\text { Gehan et al., } \\
2017\end{array}$ & https://github.com/danforthcenter/plantcv & $\begin{array}{l}\text { Object detection and } \\
\text { feature extraction; } \\
\text { spectral analysis }\end{array}$ & Python package & $\begin{array}{l}\text { Plant phenotyping } \\
\text { library }\end{array}$ & $\begin{array}{l}\text { Signal processing, } \\
\text { classic machine } \\
\text { learning }\end{array}$ \\
\hline 2017 & Trackdem & $\begin{array}{l}\text { Bruijning et al., } \\
2018\end{array}$ & https://github.com/marjoleinbruijning/trackdem & $\begin{array}{l}\text { Motion tracking and } \\
\text { blob counting }\end{array}$ & R package & $\begin{array}{l}\text { Behavioral analysis } \\
\text { pipeline }\end{array}$ & Signal processing \\
\hline 2016 & Scan-o-matic & $\begin{array}{l}\text { Zackrisson } \\
\text { et al., } 2016\end{array}$ & https://github.com/Scan-o-Matic/scanomatic & $\begin{array}{l}\text { Object detection and } \\
\text { feature extraction }\end{array}$ & Python package & $\begin{array}{l}\text { Microbial phenotyping } \\
\text { platform }\end{array}$ & Signal processing \\
\hline 2015 & MotionMeerkat & $\begin{array}{l}\text { Weinstein, } \\
2015\end{array}$ & https://github.com/bw4sz/DeepMeerkat & Motion tracking & $\begin{array}{l}\text { Python package/ } \\
\text { standalone }\end{array}$ & $\begin{array}{l}\text { Deep learning driven } \\
\text { motion detection }\end{array}$ & $\begin{array}{l}\text { Signal processing, } \\
\text { deep learning }\end{array}$ \\
\hline 2012 & ImageJ & $\begin{array}{l}\text { Schindelin } \\
\text { et al., } 2012\end{array}$ & $\begin{array}{l}\text { https://fiji.sc/; } \\
\text { https://imagej.nih.gov/ij/download.html }\end{array}$ & Multi purpose & Standalone & $\begin{array}{l}\text { Comprehensive, } \\
\text { multi-purpose image } \\
\text { processing library }\end{array}$ & $\begin{array}{l}\text { Manual processing, } \\
\text { signal processing, } \\
\text { classic machine } \\
\text { learning, feature } \\
\text { extraction }\end{array}$ \\
\hline 2003 & WingMachine & $\begin{array}{l}\text { Houle et al., } \\
2003\end{array}$ & https://www.bio.fsu.edu/\$ $\$$ sim $\$$ dhoule/Software/ & $\begin{array}{l}\text { Keypoint and feature } \\
\text { detection }\end{array}$ & Standalone & $\begin{array}{l}\text { Drosophila wing } \\
\text { morphometrics }\end{array}$ & $\begin{array}{l}\text { Signal processing, } \\
\text { feature extraction }\end{array}$ \\
\hline
\end{tabular}

Although typically first developed for a particular study system or organism (e.g., PlantCV or WorMachine), most CV applications apply techniques that are generally applicable to any type of phenotypic data contained in digital images. 
For large datasets, images with low SNR, or if the information of interest is variable across images (e.g., traits are photographed from different angles or partially covered up), machine learning approaches are probably more suitable. In contrast to signal processing, where segmentation results are immediately available, all machine learning image analysis pipelines include iterative training and validation phases, followed by a final testing phase. Such a workflow can be complex to initiate, but pays off in the long run by providing results that become increasingly robust if more training data is supplied over time. Classic machine learning algorithms often require an intermediate amount of training data (500-1,000 or more images) before they can produce satisfactory results (Schneider et al., 2020a). In this category, SVM or HOG algorithms are a good choice when areas of interest do not contrast sufficiently from the surrounding area, for example, when automatically detecting landmarks (Porto and Voje, 2020). Deep learning algorithms require much larger training datasets (minimum of 1,000-10,000 images), but are less sensitive to noise and idiosyncrasies of the foreground. Thus, for large and continuously growing data sets, or for recurring image analysis tasks, deep learning has become the standard approach for segmentation (Sultana et al., 2020). Deeper networks may increase model accuracy, and thus improve the segmentation results, but have an increasing risk of overfitting the contained information - i.e., the model is less generalizable to input data. Still, while the implementation of deep learning pipelines may require more expertise than other CV-techniques, they can be retrained and are typically less domain specific than classic machine learning pipelines (O’Mahony et al., 2020).

\section{RECENT EXAMPLES OF COMPUTER VISION TO COLLECT PHENOMIC DATA}

"Phenomics" as a term has not yet gained widespread attention in the ecological and evolutionary biology research communities (Figure 1), but many biologists are engaged in research programs that are collecting phenomic data, even though it is not called as such. Some of them are already using automatic or semiautomatic CV to collect phenotypic data. Here we present small a selection of promising applications of CV to answer ecological or evolutionary research questions (the following paragraphs are matching the panels in Figure 6).

\section{A) Shape and Texture of Resource Competition Traits}

Species diversity within ecological communities is often thought to be governed by competition for limiting resources (Chesson, 2000). However, the exact traits that make species or individuals the best competitors under resource limitation conditions are difficult to identify among all other traits. In this example, the phenotypic space underlying resource competition was explored by implementing different limitation scenarios for experimental phytoplankton communities. Images were taken with a plate reader that used a combination of visible light and fluorometry measurements (Hense et al., 2008). The images were analyzed using phenopype (Table 2; Lürig, 2021), which allowed the rapid segmentation of several 1,000 images by combining information from multiple fluorescence emission excitation spectra to an image stack. As a result, over 100 traits related to morphology (shape, size, and texture) and internal physiology (pigment content, distribution of pigments within each cell) were obtained at the individual cell level (Gallego et al., unpublished data).

\section{B) Thermal Adaptation and Thermal Reaction Norms}

Variation in body temperature can be an important source of fitness variation (Kingsolver and Huey, 2008; Svensson et al., 2020). Quantifying body temperature and thermal reaction norms in response to natural and sexual selection allows us to test predictions from evolutionary theory about phenotypic plasticity and canalization (Lande, 2009; Chevin et al., 2010). However, body temperature is an internal physiological trait that is difficult to quantify in a non-invasive way on many individuals simultaneously and under natural conditions. Thermal imaging is an efficient and non-invasive method to quantify such physiological phenotypes on a large scale and can be combined with thermal loggers to measure local thermal environmental conditions in the field (Svensson and Waller, 2013; Svensson et al., 2020).

\section{C) Stochastically Patterned Morphological Traits}

In contrast to homologous, landmark-based morphological traits, tissues also form emergent patterns that are unique to every individual. The arrangement of veins on the wings of damselflies is one such example. By measuring the spacing, angles, and connectivities within the adult wing tissue, researchers have proposed hypotheses about the mechanisms of wing development and physical constraints on wing evolution (Hoffmann et al., 2018; Salcedo et al., 2019).

\section{D) Morphometrics and Shape of Complex Structures}

Landmark-based morphometrics has become a popular tool used to characterize morphological variation in complex biological structures. Despite its popularity, landmark data is still collected mainly through manual annotation, a process which represents a significant bottleneck for phenomic studies. However, machinelearning-based CV can be used to accurately automate landmark data collection in morphometric studies not only in 2D (McPeek et al., 2008; Porto and Voje, 2020), but also in 3D (Porto et al., 2020).

\section{E) Volumes of Morphologically Complex Traits}

Many topics in evolutionary ecology concerns investment of resources into a particular trait. However, measuring energetic investment, either as mass or volume of the target traits, has been challenging because many traits are morphologically complex, making it difficult to estimate investment from a combination of linear measurements. Photogrammetry is a low-cost and fast 
technique to create $3 \mathrm{D}$ surface images from a set of images. Using a simple protocol and a free trial version of proprietary software, Tsuboi et al. (2020) demonstrated that photogrammetry can accurately measure the volume of antlers in deer family Cervidae. The protocol is still relatively low-throughput due primarily to the necessity of high number of images $(>50)$ per sample, but it allows extensive sampling [sensu (Houle et al., 2010)] of linear, area and volumetric measurements of antler structures.

\section{OUTLOOK}

In this review we provided a broad overview of various $\mathrm{CV}$ techniques and gave some recent examples of their application in ecological and evolutionary research. We presented $\mathrm{CV}$ as a promising toolkit to overcome the image analysis bottleneck in phenomics. However, to be clear, we do not suggest that biologists discontinue the collection of univariate traits like body size or discrete colors. Such measures are undoubtedly useful, if they contain explanatory value and predictive power. Instead, we propose that $\mathrm{CV}$ can help to (i) collect them with higher throughput, (ii) in a more reproducible fashion, and to (iii) collect additional traits so we can interpret them in the context of trait combinations. We argue that $\mathrm{CV}$ is not bound to immediately replace existing methods, but it simply opens the opportunity to place empirical research of phenotypes on a broader base. We also note that $\mathrm{CV}$ based phenomics can be pursued in a deductive or inductive fashion. In the former case, scientists would simply conduct hypothesis driven research including a wider array of traits into causal models (Houle et al., 2011); in the latter, they would engage in discovery-based data-mining approaches that allow scientists to form hypotheses a posteriori based on the collected data (Kell and Oliver, 2004).

Although CV based phenomics provides new opportunities for many areas of study, we identify several specific fields that will profit most immediately from CV. First, evolutionary quantitative genetics will benefit tremendously from increased sample sizes that CV-phenomics entails, because the bottleneck of the field has been the difficulty in accurately estimating key statistics such as genetic variance covariance matrices and selection gradients. The recent discovery of tight matches between mutational, genetic, and macroevolutionary variances in Drosophilid wing shape (Houle et al., 2017) is exemplary of a successful phenomic project. Second, large-scale empirical studies of the genotype-phenotype map will finally become possible, because of the availability of high-throughput phenotypic data and analytical framework to deal with big data (Pitchers et al., 2019; Zheng et al., 2019; Maeda et al., 2020). Third, studies of fossil time-series will gain opportunities to document and analyze the dynamics of long-term phenotypic evolution with unprecedented temporal resolution (Brombacher et al., 2017; Liow et al., 2017). The ever-growing technology of $\mathrm{CV}$ indicates that these are likely a small subset of unforeseen future applications of $\mathrm{CV}$ phenomics in our field. Similar to the technological advancements in DNA-sequencing that have revolutionized our view of genomes, development, and molecular evolution in the past decades, we anticipate that the way we look at phenotypic data will be changing in the coming years.

Just as $\mathrm{CV}$ is changing what it means to measure a trait, there is a complementary change in what can be considered scientific image data in the first place. Large, publicly available image datasets are fertile ground for ecology and evolutionary research. Such databases include both popular and non-scientific social media (e.g., Flickr or Instagram), but also qualitycontrolled and vetted natural history and species identification resources with global scope and ambitions (e.g., iNaturalist). Successful examples of how such public image databases can be useful are in studies aiming to quantify the frequencies variation of discrete traits, such as color polymorphism frequencies in different geographic regions (Leighton et al., 2016). These manual efforts in mining available public image resources can potentially be replaced in the future using more automated machine learning or CV approaches. Similarly, the corpus of published scientific literature is full of image data that can be combined and re-analyzed in order to address larger-scale questions (Hoffmann et al., 2018; Church et al., 2019a,b).

Previous calls for phenomics argued that, to make phenomics a successful endeavour, it has to be extensive, aiming at measuring many different aspects of the phenotypes, as well as intensive, aiming at characterizing each measurement accurately with large sample size and with high temporal resolution (Bilder et al., 2009; Houle et al., 2010; Furbank and Tester, 2011). We agree with this view, but we also emphasize that phenomics is nothing conceptually new in this respect. As discussed above, many researchers in our field have already adopted phenomic pipelines, i.e., they are collecting high-dimensional phenotypic data on a large scale, but they may not be using the term "phenomics." If so, what is the conceptual value and added benefit of explicitly studying phenomes? We argue that $\mathrm{CV}$ and other techniques will facilitate the rigorous quantification of phenomes in the same fashion as next generation genomics allows scientists to move away from a few markers of interest to simply reading all molecular data that is available. While it may not be possible to extract phenomes in a complete fashion (Houle et al., 2010), a "phenomics-mindset" still gives us the opportunity to collect and analyze larger amounts of phenotypic data with virtually no extra cost. Taking images and analyzing them with $\mathrm{CV}$ enables biologists to choose freely between conducting conventional research of phenotypes, but with higher throughput and in a reproducible fashion, or to truly "harness the power big data" (Peters et al., 2014) for the study of high dimensional phenotypic data.

\section{AUTHOR CONTRIBUTIONS}

ML conceived the idea for this review and initiated its writing. In the process, all authors contributed equally to the development and discussion of ideas, and to the writing of the manuscript. 


\section{FUNDING}

The publication of this study was funded through the Swedish Research Council International Postdoc Grant (2016-06635) to MT. ML was supported by a Swiss

\section{REFERENCES}

Alom, M. Z., Taha, T. M., Yakopcic, C., Westberg, S., Sidike, P., Nasrin, M. S., et al. (2018). The history began from AlexNet: a comprehensive survey on deep learning approaches. arXiv [cs.CV]. [Preprint]. arXiv:1803.01164.

Bateson, A., and Curtiss, B. (1996). A method for manual endmember selection and spectral unmixing. Remote Sens. Environ. 55, 229-243. doi: 10.1016/S00344257(95)00177-8

Bauer, E., and Kohavi, R. (1999). An empirical comparison of voting classification algorithms: bagging, boosting, and variants. Mach. Learn. 36, 105-139. doi: 10.1023/A:1007515423169

Bertin, E., Marcelpoil, R., and Chassery, J.-M. (1992). "Morphological algorithms based on Voronoi and Delaunay graphs: microscopic and medical applications," in Proceedings of the Image Algebra and Morphological Image Processing III, (Bellingham, WA: International Society for Optics and Photonics), 356-367. doi: $10.1117 / 12.60655$

Beucher, S. (1979). "Use of watersheds in contour detection," in Proceedings of the International Workshop on Image. Available online at: https://ci.nii.ac.jp/naid/ 10008961959/

Bilder, R. M., Sabb, F. W., Cannon, T. D., London, E. D., Jentsch, J. D., Parker, D. S., et al. (2009). Phenomics: the systematic study of phenotypes on a genome-wide scale. Neuroscience 164, 30-42. doi: 10.1016/j.neuroscience.2009.01.027

Blonder, B. (2018). Hypervolume concepts in niche- and trait-based ecology. Ecography 41, 1441-1455. doi: 10.1111/ecog.03187

Breiman, L. (2001). Random forests. Mach. Learn. 45, 5-32. doi: 10.1023/A: 1010933404324

Brombacher, A., Wilson, P. A., Bailey, I., and Ezard, T. H. G. (2017). The breakdown of static and evolutionary allometries during climatic upheaval. Am. Nat. 190, 350-362. doi: 10.1086/692570

Bruijning, M., Visser, M. D., Hallmann, C. A., and Jongejans, E. (2018). trackdem : automated particle tracking to obtain population counts and size distributions from videos in r. Methods Ecol. Evol. 9, 965-973. doi: 10.1111/2041-210X. 12975

Buetti-Dinh, A., Galli, V., Bellenberg, S., Ilie, O., Herold, M., Christel, S., et al. (2019). Deep neural networks outperform human expert's capacity in characterizing bioleaching bacterial biofilm composition. Biotechnol. Rep. (Amst) 22:e00321. doi: 10.1016/j.btre.2019. e00321

Canny, J. (1986). A computational approach to edge detection. IEEE Trans. Pattern Anal. Mach. Intell. PAMI 8, 679-698. doi: 10.1109/TPAMI.1986.4767851

Cheng, K. C., Xin, X., Clark, D. P., and La Riviere, P. (2011). Whole-animal imaging, gene function, and the zebrafish phenome project. Curr. Opin. Genet. Dev. 21, 620-629. doi: 10.1016/j.gde.2011.08.006

Chesson, P. (2000). Mechanisms of maintenance of species diversity. Annu. Rev. Ecol. Syst. 31, 343-366. doi: 10.1146/annurev.ecolsys.31.1.343

Chevin, L.-M., Lande, R., and Mace, G. M. (2010). Adaptation, plasticity, and extinction in a changing environment: towards a predictive theory. PLoS Biol. 8:e1000357. doi: 10.1371/journal.pbio.1000357

Church, G. M., and Gilbert, W. (1984). Genomic sequencing. Proc. Natl. Acad. Sci. U.S.A. 81, 1991-1995. doi: 10.1073/pnas.81.7.1991

Church, S. H., Donoughe, S., de Medeiros, B. A. S., and Extavour, C. G. (2019a). A dataset of egg size and shape from more than 6,700 insect species. Sci. Data 6:104. doi: 10.1038/s41597-019-0049-y

Church, S. H., Donoughe, S., de Medeiros, B. A. S., and Extavour, C. G. (2019b). Insect egg size and shape evolve with ecology but not developmental rate. Nature 571, 58-62. doi: 10.1038/s41586-019-1302-4

Cortes, C., and Vapnik, V. (1995). Support-vector networks. Mach. Learn. 20, 273-297. doi: 10.1023/A:1022627411411

Dalal, N., and Triggs, B. (2005). "Histograms of oriented gradients for human detection," in Proceedings of the 2005 IEEE Computer Society Conference on
National Science Foundation Early Postdoc. Mobility grant (SNSF: P2EZP3_191804). ES was funded by a grant from the Swedish Research Council (VR: Grant No. 201603356). SD was supported by the Jane Coffin Childs Memorial Fund.

Computer Vision and Pattern Recognition (CVPR'05), Vol. 1, San Diego, CA, 886-893. doi: 10.1109/CVPR.2005.177

Di, Z., Klop, M. J. D., Rogkoti, V.-M., Le Dévédec, S. E., van de Water, B., Verbeek, F. J., et al. (2014). Ultra high content image analysis and phenotype profiling of 3D cultured micro-tissues. PLoS One 9:e109688. doi: 10.1371/journal.pone. 0109688

Dietterich, T. G. (2000). "Ensemble methods in machine learning," in Proceedings of the First International Workshop on Multiple Classifier Systems MCS '00, (Berlin: Springer-Verlag), 1-15. doi: 10.1142/9789811201967_0001

Duda, R. O., and Hart, P. E. (1972). Use of the Hough transformation to detect lines and curves in pictures. Commun. ACM 15, 11-15. doi: 10.1145/361237.361242

Edgington, D. R., Cline, D. E., Davis, D., Kerkez, I., and Mariette, J. (2006). Detecting, tracking and classifying animals in underwater video. OCEANS 2006, 1-5. doi: 10.1109/OCEANS.2006.306878

Feder, M. E., and Mitchell-Olds, T. (2003). Evolutionary and ecological functional genomics. Nat. Rev. Genet. 4, 651-657. doi: 10.1038/nrg1128

Fossum, E. R., and Hondongwa, D. B. (2014). A review of the pinned photodiode for CCD and CMOS image sensors. IEEE J. Electron Devices Soc. 2, 33-43. doi: $10.1109 /$ jeds.2014.2306412

Freimer, N., and Sabatti, C. (2003). The human phenome project. Nat. Genet. 34, 15-21. doi: 10.1038/ng0503-15

French, S., Coutts, B. E., and Brown, E. D. (2018). Open-source high-throughput phenomics of bacterial promoter-reporter strains. Cell Syst 7, 339-346.e3. doi: 10.1016/j.cels.2018.07.004

Friedman, J. H. (2000). Greedy function approximation: a gradient boosting machine. Ann. Stat. 29, 1189-1232.

Furbank, R. T., and Tester, M. (2011). Phenomics-technologies to relieve the phenotyping bottleneck. Trends Plant Sci. 16, 635-644. doi: 10.1016/j.tplants. 2011.09.005

Gehan, M. A., Fahlgren, N., Abbasi, A., Berry, J. C., Callen, S. T., Chavez, L., et al. (2017). PlantCV v2: image analysis software for high-throughput plant phenotyping. PeerJ 5:e4088. doi: 10.7717/peerj.4088

Gerum, R. C., Richter, S., Fabry, B., and Zitterbart, D. P. (2017). ClickPoints: an expandable toolbox for scientific image annotation and analysis. Methods Ecol. Evol. 8, 750-756. doi: 10.1111/2041-210X.12702

Goesele, M. (2004). New Acquisition Techniques for Real Objects and Light Sources in Computer Graphics. Norderstedt: Books on Demand.

Grabowski, M., and Porto, A. (2017). How many more? Sample size determination in studies of morphological integration and evolvability. Methods Ecol. Evol. 8, 592-603. doi: 10.1111/2041-210X.12674

Greenspan, H., Ruf, A., and Goldberger, J. (2006). Constrained Gaussian mixture model framework for automatic segmentation of MR brain images. IEEE Trans. Med. Imaging 25, 1233-1245. doi: 10.1109/tmi.2006.880668

Hakim, A., Mor, Y., Toker, I. A., Levine, A., Neuhof, M., Markovitz, Y., et al. (2018). WorMachine: machine learning-based phenotypic analysis tool for worms. BMC Biol. 16:8. doi: 10.1186/s12915-017-0477-0

Heaton, J. (2020). Applications of deep neural networks. arXiv [cs.LG]. [Preprint]. arXiv:2009.05673.

Heileman, G. L., and Myler, H. R. (1989). Theoretical and Experimental Aspects of Supervised Learning in Artificial Neural Networks. Available online at: https: //dl.acm.org/citation.cfm?id=915701

Hense, B. A., Gais, P., Jutting, U., Scherb, H., and Rodenacker, K. (2008). Use of fluorescence information for automated phytoplankton investigation by image analysis. J. Plankton Res. 30, 587-606. doi: 10.1093/plankt/fbn024

Hinton, G., and Sejnowski, T. J. (1999). Unsupervised Learning: Foundations of Neural Computation. Cambridge, MA: MIT Press.

Hoffmann, J., Donoughe, S., Li, K., Salcedo, M. K., and Rycroft, C. H. (2018). A simple developmental model recapitulates complex insect wing venation patterns. Proc. Natl. Acad. Sci. U.S.A. 115, 9905-9910. doi: 10.1073/pnas. 1721248115 
Hooper, D. U., Chapin, F. S. III, Ewel, J. J., Hector, A., Inchausti, P., Lavorel, S., et al. (2005). EFFECTS OF BIODIVERSITY ON ECOSYSTEM FUNCTIONING: A CONSENSUS OF CURRENT KNOWLEDGE. Ecol. Monogr. 75, 3-35. doi: 10.1890/04-0922

Houle, D., Bolstad, G. H., van der Linde, K., and Hansen, T. F. (2017). Mutation predicts 40 million years of fly wing evolution. Nature 548, 447-450. doi: 10. 1038/nature23473

Houle, D., Govindaraju, D. R., and Omholt, S. (2010). Phenomics: the next challenge. Nat. Rev. Genet. 11, 855-866. doi: 10.1038/nrg2897

Houle, D., Mezey, J., Galpern, P., and Carter, A. (2003). Automated measurement of Drosophila wings. BMC Evol. Biol. 3:25. doi: 10.1186/1471-2148-3-25

Houle, D., Pélabon, C., Wagner, G. P., and Hansen, T. F. (2011). Measurement and meaning in biology. Q. Rev. Biol. 86, 3-34. doi: 10.1086/658408

Høye, T. T., Ärje, J., Bjerge, K., Hansen, O. L. P., Iosifidis, A., Leese, F., et al. (2020). Deep learning and computer vision will transform entomology. Ecology 118:e2002545117. doi: 10.1101/2020.07.03.187252

Hsiang, A. Y., Nelson, K., Elder, L. E., Sibert, E. C., Kahanamoku, S. S., Burke, J. E., et al. (2018). AutoMorph : accelerating morphometrics with automated 2D and 3D image processing and shape extraction. Methods Ecol. Evol. 9, 605-612. doi: 10.1111/2041-210X.12915

$\mathrm{Hu}$, Q., and Davis, C. (2005). Automatic plankton image recognition with cooccurrence matrices and Support Vector Machine. Mar. Ecol. Prog. Ser. 295, 21-31. doi: 10.3354/meps295021

Ishikawa, A., Kabeya, N., Ikeya, K., Kakioka, R., Cech, J. N., Osada, N., et al. (2019). A key metabolic gene for recurrent freshwater colonization and radiation in fishes. Science 364, 886-889. doi: 10.1126/science.aau5656

Kanopoulos, N., Vasanthavada, N., and Baker, R. L. (1988). Design of an image edge detection filter using the Sobel operator. IEEE J. Solid-State Circuits 23, 358-367. doi: $10.1109 / 4.996$

Kell, D. B., and Oliver, S. G. (2004). Here is the evidence, now what is the hypothesis? The complementary roles of inductive and hypothesis-driven science in the post-genomic era. Bioessays 26, 99-105. doi: 10.1002/bies.10385

Kingsolver, J. G., and Huey, R. B. (2008). Size, temperature, and fitness: three rules. Evol. Ecol. Res. 10, 251-268.

Krizhevsky, A., Sutskever, I., and Hinton, G. E. (2012). "ImageNet classification with deep convolutional neural networks," in Advances in Neural Information Processing Systems 25, eds F. Pereira, C. J. C. Burges, L. Bottou, and K. Q. Weinberger (New York, NY: Curran Associates, Inc), 1097-1105.

Sollich, P. and Krogh, A. (1996). "Learning with ensembles: how over-fitting can be useful," in Proceedings of the 1995 Conference, 190, Cambridge, MA.

Kühl, H. S., and Burghardt, T. (2013). Animal biometrics: quantifying and detecting phenotypic appearance. Trends Ecol. Evol. 28, 432-441. doi: 10.1016/ j.tree.2013.02.013

Lamichhaney, S., Card, D. C., Grayson, P., Tonini, J. F. R., Bravo, G. A., Näpflin, K., et al. (2019). Integrating natural history collections and comparative genomics to study the genetic architecture of convergent evolution. Philos. Trans. R. Soc. Lond. B Biol. Sci. 374:20180248. doi: 10.1098/rstb.2018.0248

Lande, R. (2009). Adaptation to an extraordinary environment by evolution of phenotypic plasticity and genetic assimilation. J. Evol. Biol. 22, 1435-1446. doi: 10.1111/j.1420-9101.2009.01754.x

Lande, R., and Arnold, S. J. (1983). The measurement of selection on correlated characters. Evolution 37, 1210-1226. doi: 10.2307/2408842

Laughlin, D. C., Gremer, J. R., Adler, P. B., Mitchell, R. M., and Moore, M. M. (2020). The net effect of functional traits on fitness. Trends Ecol. Evol. 35, 1037-1047. doi: 10.1016/j.tree.2020.07.010

Le, V.-L., Beurton-Aimar, M., Zemmari, A., Marie, A., and Parisey, N. (2020). Automated landmarking for insects morphometric analysis using deep neural networks. Ecol. Inform. 60:101175. doi: 10.1016/j.ecoinf.2020.101175

LeCun, Y., Bengio, Y., and Hinton, G. (2015). Deep learning. Nature 521, 436-444. doi: $10.1038 /$ nature 14539

Leighton, G., Hugo, P. S., Roulin, A., and Amar, A. (2016). Just Google it: assessing the use of Google Images to describe geographical variation in visible traits of organisms. Br. Ecol. Soc. 7, 1060-1070. doi: 10.1111/2041-210X.12562

Liow, L. H., Di Martino, E., Krzeminska, M., Ramsfjell, M., Rust, S., Taylor, P. D., et al. (2017). Relative size predicts competitive outcome through 2 million years. Ecol. Lett. 20, 981-988. doi: 10.1111/ele.12795

Liu, F., Wollstein, A., Hysi, P. G., Ankra-Badu, G. A., Spector, T. D., Park, D., et al. (2010). Digital quantification of human eye color highlights genetic association of three new loci. PLoS Genetics 6:e1000934. doi: 10.1371/journal. pgen. 1000934

Lloyd, S. (1982). Least squares quantization in PCM. IEEE Trans. Inf. Theory 28, 129-137. doi: 10.1109/TIT.1982.1056489

Lowe, D. G. (1999). "Object recognition from local scale-invariant features," in Proceedings of the Seventh IEEE International Conference on Computer Vision, Vol. 2, Kerkyra, 1150-1157. doi: 10.1109/ICCV.1999.790410

Lowe, D. G. (2004). Distinctive image features from scale-invariant keypoints. Int. J. Comput. Vis. 60, 91-110. doi: 10.1023/b:visi.0000029664.99615.94

Lürig, M. D. (2021). phenopype: a phenotyping pipeline for Python. Cold Spring Harb. Lab. bioRxiv. doi: 10.1101/2021.03.17.435781

Lytle, D. A., Martínez-Muñoz, G., Zhang, W., Larios, N., Shapiro, L., Paasch, R., et al. (2010). Automated processing and identification of benthic invertebrate samples. J. North Am. Benthol. Soc. 29, 867-874. doi: 10.1899/09-080.1

Maeda, T., Iwasawa, J., Kotani, H., Sakata, N., Kawada, M., Horinouchi, T., et al. (2020). High-throughput laboratory evolution reveals evolutionary constraints in Escherichia coli. Nat. Commun. 11:5970. doi: 10.1038/s41467-020-19713-w

Mäkelä, T., Clarysse, P., Sipilä, O., Pauna, N., Pham, Q. C., Katila, T., et al. (2002). A review of cardiac image registration methods. IEEE Trans. Med. Imaging 21, 1011-1021. doi: 10.1109/TMI.2002.804441

McPeek, M. A., Shen, L., Torrey, J. Z., and Farid, H. (2008). The tempo and mode of three-dimensional morphological evolution in male reproductive structures. Am. Nat. 171, E158-E178. doi: 10.1086/587076

McQuin, C., Goodman, A., Chernyshev, V., Kamentsky, L., Cimini, B. A., Karhohs, K. W., et al. (2018). CellProfiler 3.0: next-generation image processing for biology. PLoS Biol. 16:e2005970. doi: 10.1371/journal.pbio.2005970

Minsky, M. (1961). Steps toward Artificial Intelligence. Proc. IRE 49, 8-30. doi: 10.1109/JRPROC.1961.287775

Minsky, M., and Papert, S. (1969). Perceptrons. Available online at: http://papers. cumincad.org/cgi-bin/works/Show?_id=b029 (accessed December 7, 2020).

Mitchell, T. M. (1997). Machine Learning. 1997, Vol. 45. Burr Ridge, IL: McGraw Hill, 870-877.

Morel-Journel, T., Thuillier, V., Pennekamp, F., Laurent, E., Legrand, D., Chaine, A. S., et al. (2020). A multidimensional approach to the expression of phenotypic plasticity. Funct. Ecol. 34, 2338-2349. doi: 10.1111/1365-2435. 13667

Mortensen, E. N., Delgado, E. L., Deng, H., Lytle, D., Moldenke, A., Paasch, R., et al. (2007). Pattern Recognition for Ecological Science and Environmental Monitoring: An Initial Report. Available online at: http://citeseerx.ist.psu. edu/viewdoc/download?doi=10.1.1.88.2058\&rep=rep1\&type=pdf $\quad$ (accessed February 10, 2021).

Norouzzadeh, M. S., Nguyen, A., Kosmala, M., Swanson, A., Palmer, M. S., Packer, C., et al. (2018). Automatically identifying, counting, and describing wild animals in camera-trap images with deep learning. Proc. Natl. Acad. Sci. U.S.A. 115, E5716-E5725. doi: 10.1073/pnas.1719367115

O’Mahony, N., Campbell, S., Carvalho, A., Harapanahalli, S., Hernandez, G. V., Krpalkova, L., et al. (2020). Deep learning vs. traditional computer vision. Adv. Intell. Syst. Comput. 128-144. doi: 10.1007/978-3-030-17795-9_10

Orgogozo, V., Morizot, B., and Martin, A. (2015). The differential view of genotype-phenotype relationships. Front. Genet. 6:179. doi: 10.3389/fgene.2015 00179

Petchey, O. L., and Gaston, K. J. (2006). Functional diversity: back to basics and looking forward. Ecol. Lett. 9, 741-758. doi: 10.1111/j.1461-0248.2006.00924.x

Peters, D. P. C., Havstad, K. M., Cushing, J., Tweedie, C., Fuentes, O., and Villanueva-Rosales, N. (2014). Harnessing the power of big data: infusing the scientific method with machine learning to transform ecology. Ecosphere 5, 1-15. doi: 10.1890/ES13-00359.1

Pfennig, D. W., Wund, M. A., Snell-Rood, E. C., Cruickshank, T., Schlichting, C. D., and Moczek, A. P. (2010). Phenotypic plasticity's impacts on diversification and speciation. Trends Ecol. Evol. 25, 459-467. doi: 10.1016/j. tree.2010.05.006

Phillips, P. C., and Arnold, S. J. (1999). Hierarchical comparison of genetic variance-covariance matrices. I. using the flury hierarchy. Evolution 53, 15061515. doi: $10.2307 / 2640896$

Piccardi, M. (2004). "Background subtraction techniques: a review," in Proceedings of the 2004 IEEE International Conference on Systems, Man and Cybernetics (IEEE Cat. No.04CH37583), Vol. 4, The Hague, 3099-3104. doi: 10.1109/ ICSMC.2004.1400815 
Pitchers, W., Nye, J., Márquez, E. J., Kowalski, A., Dworkin, I., and Houle, D. (2019). A multivariate genome-wide association study of wing shape in Drosophila melanogaster. Genetics 211, 1429-1447. doi: 10.1534/genetics.118. 301342

Pointer, M. R., and Attridge, G. G. (1998). The Number of Discernible Colours. Color Research \& Application: Endorsed by Inter-Society Color Council, The Colour Group (Great Britain), Canadian Society for Color, Color Science Association of Japan, Dutch Society for the Study of Color, The Swedish Colour Centre Foundation, Colour Society of Australia, Centre Français de la Couleur 23, 52-54. Available online at: https://onlinelibrary.wiley.com/doi/abs/10.1002/(SICI)1520-6378(199802)23: 1\%3C52::AID-COL8\%3E3.0.CO;2-2?casa_token=7LmOYEdzguoAAAAA: pg8hhYjlLpw6USMpbyZwgK8ZEt2mF6AtsPDy_82LdaU-15tzK6I-XheHSb_ 6ejjUumAQgwG8MJu_CT4

Porto, A., and Lysne Voje, K. (2020). ML-morph: a fast, accurate and general approach for automated detection and landmarking of biological structures in images. Methods Ecol. Evol. 11, 500-512. doi: 10.1111/2041-210X.13373

Porto, A., Rolfe, S. M., and Murat Maga, A. (2020). ALPACA: a fast and accurate approach for automated landmarking of three-dimensional biological structures. Cold Spring Harb. Lab. bioRxiv. doi: 10.1101/2020.09.18.303891

Reynolds, D. A., and Rose, R. C. (1995). Robust text-independent speaker identification using Gaussian mixture speaker models. IEEE Trans. Audio Speech Lang. Process. 3, 72-83. doi: 10.1109/89.365379

Ringnér, M. (2008). What is principal component analysis? Nat. Biotechnol. 26, 303-304. doi: 10.1038/nbt0308-303

Roberts, L. G. (1963). Machine Perception of Three-Dimensional Solids. Available online at: https://dspace.mit.edu/handle/1721.1/11589?show=full (accessed December 7, 2020).

Rodenacker, K., Brühl, A., Hausner, M., Kühn, M., Liebscher, V., Wagner, M., et al. (2000). Quantification of biofilms in multi-spectral digitall volumes from confocal laser-scanning microscopes. Image Anal. Stereol. 19:151. doi: 10.5566/ ias.v19.p151-156

Roeder, A. H. K., Cunha, A., Burl, M. C., and Meyerowitz, E. M. (2012). A computational image analysis glossary for biologists. Development 139, 30713080. doi: $10.1242 / \mathrm{dev} .076414$

Rosenblatt, F. (1958). The perceptron: a probabilistic model for information storage and organization in the brain. Psychol. Rev. 65, 386-408. doi: 10.1037/ h0042519

Rosten, E., and Drummond, T. (2006). Machine Learning for High-Speed Corner Detection. in Computer Vision - ECCV 2006. Berlin Heidelberg: Springer, 430-443. doi: 10.1007/11744023_34

Rumelhart, D. E., and McClelland, J. L. (eds) (1987). "Learning internal representations by error propagation," in Parallel Distributed Processing: Explorations in the Microstructure of Cognition: Foundations. Cambridge, MA: MIT Press, 318-362.

Salcedo, M. K., Hoffmann, J., Donoughe, S., and Mahadevan, L. (2019). Computational analysis of size, shape and structure of insect wings. Biol. Open 8:bio04077. doi: 10.1242/bio.040774

Saltz, J. B., Hessel, F. C., and Kelly, M. W. (2017). Trait correlations in the genomics era. Trends Ecol. Evol. 32, 279-290. doi: 10.1016/j.tree.2016.12.008

Sanchez-Hernandez, C., Boyd, D. S., and Foody, G. M. (2007). Mapping specific habitats from remotely sensed imagery: support vector machine and support vector data description based classification of coastal saltmarsh habitats. Ecol. Inform. 2, 83-88. doi: 10.1016/j.ecoinf.2007.04.003

Schindelin, J., Arganda-Carreras, I., Frise, E., Kaynig, V., Longair, M., Pietzsch, T., et al. (2012). Fiji: an open-source platform for biological-image analysis. Nat. Methods 9, 676-682. doi: 10.1038/nmeth.2019

Schluter, D. (1996). Adaptive radiation along genetic lines of least resistance. Evolution 50, 1766-1774. doi: 10.1111/j.1558-5646.1996.tb03563.x

Schneider, S., Greenberg, S., Taylor, G. W., and Kremer, S. C. (2020a). Three critical factors affecting automated image species recognition performance for camera traps. Ecol. Evol. 10, 3503-3517. doi: 10.1002/ece3.6147

Schneider, S., Taylor, G. W., and Kremer, S. C. (2020b). "Similarity learning networks for animal individual re-identification-beyond the capabilities of a human observer," in Proceedings of the IEEE/CVF Winter Conference on Applications of Computer Vision Workshops, Snowmass, CO, $44-52$.
Seehausen, O., Butlin, R. K., Keller, I., Wagner, C. E., Boughman, J. W., Hohenlohe, P. A., et al. (2014). Genomics and the origin of species. Nat. Rev. Genet. 15, 176-192. doi: 10.1038/nrg3644

Shapiro, L. G., and Stockman, G. C. (2001). Computer Vision. Upper Saddle River, NJ: Prentice Hall.

Shorten, C., and Khoshgoftaar, T. M. (2019). A survey on image data augmentation for deep learning. J. Big Data 6:60. doi: 10.1186/s40537-019-0197-0

Sinervo, B., and Svensson, E. (2002). Correlational selection and the evolution of genomic architecture. Heredity 89, 329-338. doi: 10.1038/sj.hdy.6800148

Soulé, M. (1967). PHENETICS OF NATURAL POPULATIONS I. PHENETIC RELATIONSHIPS OF INSULAR POPULATIONS OF THE SIDEBLOTCHED LIZARD. Evolution 21, 584-591. doi: 10.1111/j.1558-5646.1967.tb 03413.x

Sultana, F., Sufian, A., and Dutta, P. (2020). Evolution of image segmentation using deep convolutional neural network: a survey. arXiv [cs.CV]. [Preprint]. arXiv:2001.04074.

Svensson, E. I., Arnold, S. J., Bürger, R., Csilléry, K., Draghi, J., Henshaw, J. M., et al. (2021). Correlational selection in the age of genomics. Nat. Ecol. Evol. (in press).

Svensson, E. I., Gomez-Llano, M., and Waller, J. T. (2020). Selection on phenotypic plasticity favors thermal canalizatio. Proc. Nat. Acad. Sci. 117, 29767-29774. doi: 10.1073/pnas.2012454117

Svensson, E. I., and Waller, J. T. (2013). Ecology and sexual selection: evolution of wing pigmentation in calopterygid damselflies in relation to latitude, sexual dimorphism, and speciation. Am. Nat. 182, E174-E195. doi: 10.1086/ 673206

Tattersall, G. J., Andrade, D. V., and Abe, A. S. (2009). Heat exchange from the toucan bill reveals a controllable vascular thermal radiator. Science 325 , 468-470. doi: 10.1126/science.1175553

Tattersall, G. J., and Cadena, V. (2010). Insights into animal temperature adaptations revealed through thermal imaging. Imaging Sci. J. 58, 261-268. doi: 10.1179/136821910X12695060594165

Tsubaki, Y., Samejima, Y., and Siva-Jothy, M. T. (2010). Damselfly females prefer hot males: higher courtship success in males in sunspots. Behav. Ecol. Sociobiol. 64, 1547-1554. doi: 10.1007/s00265-010-0968-2

Tsuboi, M., Kopperud, B. T., Syrowatka, C., Grabowski, M., Voje, K. L., Pélabon, C., et al. (2020). Measuring complex morphological traits with 3D photogrammetry: a case study with deer antlers. Evol. Biol. 47, 175-186. doi: 10.1007/s11692-020-09496-9

Turk, M., and Pentland, A. (1991). Eigenfaces for recognition. J. Cogn. Neurosci. 3, 71-86. doi: 10.1162/jocn.1991.3.1.71

Ubbens, J. R., and Stavness, I. (2017). Deep plant phenomics: a deep learning platform for complex plant phenotyping tasks. Front. Plant Sci. 8:1190. doi: 10.3389/fpls.2017.01190

Valan, M., Makonyi, K., Maki, A., Vondráèek, D., and Ronquist, F. (2019). Automated taxonomic identification of insects with expert-level accuracy using effective feature transfer from convolutional networks. Syst. Biol. 68, 876-895. doi: 10.1093/sysbio/syz014

Villéger, S., Mason, N. W. H., and Mouillot, D. (2008). New multidimensional functional diversity indices for a multifaceted framework in functional ecology. Ecology 89, 2290-2301. doi: 10.1890/07-1206.1

Visscher, P. M., and Yang, J. (2016). A plethora of pleiotropy across complex traits. Nat. Genet. 48, 707-708. doi: 10.1038/ng.3604

Wäldchen, J., and Mäder, P. (2018). Machine learning for image based species identification. Methods Ecol. Evol. 9, 2216-2225. doi: 10.1111/2041210X.13075

Walsh, B. (2007). Escape from flatland. J. Evol. Biol. 20, 36-8; discussion 39-44. doi: $10.1111 / j .1420-9101.2006 .01218 . x$

Weinstein, B. G. (2015). MotionMeerkat: integrating motion video detection and ecological monitoring. Methods Ecol. Evol. 6, 357-362. doi: 10.1111/2041-210X. 12320

Wessman, C. A., Bateson, C. A., and Benning, T. L. (1997). Detecting fire and grazing patterns in tallgrass prairie using spectral mixture analysis. Ecol. Appl. 7, 493-511. doi: 10.1890/1051-0761(1997)007[0493:dfagpi]2.0.co;2

Williams, J. B. (2017). "Electronics invades photography: digital cameras," in The Electronics Revolution: Inventing the Future, ed. J. B. Williams (Cham: Springer International Publishing), 243-250. doi: 10.1007/978-3-319-49088-5_26 
Wolak, M. E., Fairbairn, D. J., and Paulsen, Y. R. (2012). Guidelines for estimating repeatability. Methods Ecol. Evol. 3, 129-137. doi: 10.1111/j.2041-210X.2011. 00125.x

Wolman, A. G. (2006). Measurement and meaningfulness in conservation science. Conserv. Biol. 20, 1626-1634. doi: 10.1111/j.15231739.2006.00531.x

Zackrisson, M., Hallin, J., Ottosson, L.-G., Dahl, P., Fernandez-Parada, E., Ländström, E., et al. (2016). Scan-o-matic: high-resolution microbial phenomics at a massive scale. G3 6, 3003-3014. doi: 10.1534/g3.116.032342

Zhang, Y., and Wu, L. (2011). Optimal multi-level thresholding based on maximum tsallis entropy via an artificial bee colony approach. Entropy 13, 841-859. doi: 10.3390/e13040841

Zheng, J., Payne, J. L., and Wagner, A. (2019). Cryptic genetic variation accelerates evolution by opening access to diverse adaptive peaks. Science 365, 347-353. doi: $10.1126 /$ science.aax 1837
Zhou, X., and Wang, X. (2006). Optimisation of Gaussian mixture model for satellite image classification. IEE Proc. Vision Image Signal Process. 153, 349356. doi: $10.1049 /$ ip-vis:20045126

Conflict of Interest: The authors declare that the research was conducted in the absence of any commercial or financial relationships that could be construed as a potential conflict of interest.

Copyright (c) 2021 Lürig, Donoughe, Svensson, Porto and Tsuboi. This is an openaccess article distributed under the terms of the Creative Commons Attribution License (CC BY). The use, distribution or reproduction in other forums is permitted, provided the original author(s) and the copyright owner(s) are credited and that the original publication in this journal is cited, in accordance with accepted academic practice. No use, distribution or reproduction is permitted which does not comply with these terms. 